\title{
Hall algebras in the derived category and higher-rank DT invariants
}

\author{
Yukinobu Toda
}

\begin{abstract}
We remark that the combination of the works of Ben-Bassat-Brav-Bussi-Joyce and Alper-Hall-Rydh imply the conjectured local description of the moduli stacks of semiSchur objects in the derived category of coherent sheaves on projective Calabi-Yau 3 -folds. This result was assumed in the author's previous papers to apply wall-crossing formulas of DT-type invariants in the derived category, for example DT/PT correspondence, rationality, etc. We also show that the above result can be applied to prove the higher-rank version of the DT/PT correspondence and rationality.
\end{abstract}

\section{Introduction}

\subsection{Moduli stacks of semi-Schur objects on Calabi-Yau 3-folds}

The Donaldson-Thomas (DT for short) invariants were introduced by Thomas [Tho00] as holomorphic analogue of Casson invariants on real 3-manifolds. They count stable sheaves on CalabiYau 3-folds, and their rank one theory is conjectured (and proved in many cases [PP17]) to be related to Gromov-Witten invariants [MNOP06]. On the other hand, Joyce-Song [JS12] and Kontsevich-Soibelman [KS08] introduced generalized DT invariants that also count strictly semistable sheaves, and proved their wall-crossing formula. It has been expected that the wallcrossing formula is also applicable for DT-type invariants counting Bridgeland semistable objects [Bri07] or weak semistable objects [Tod10a] in the derived category of coherent sheaves. Although almost all the technical details are parallel with [JS12], there has been a technical issue, namely a certain local description of the moduli stack of semistable objects in the derived category. That technical issue was assumed in the author's previous papers [Tod10a, Tod13b, Tod11, Tod12, Tod13a, Tod16] so that the proofs of their main formulas on DT invariants were not mathematically rigorous except those for their Euler characteristic version. (The Euler characteristic version is the formula for the naive Euler characteristics of the moduli spaces of sheaves without the weight by the Behrend function.)

The first purpose of this note is to remark that the above technical issue is now settled, just by combining the works of Ben-Bassat-Brav-Bussi-Joyce [BBBJ15] and Alper-Hall-Rydh

Received 3 February 2016, accepted in final form 14 April 2019.

2010 Mathematics Subject Classification 14D20, 14J32, $18 \mathrm{E} 30$.

Keywords: Donaldson-Thomas invariants, Hall algebras, derived category.

This journal is (c) Foundation Compositio Mathematica 2020. This article is distributed with Open Access under the terms of the Creative Commons Attribution Non-Commercial License, which permits non-commercial reuse, distribution, and reproduction in any medium, provided that the original work is properly cited. For commercial re-use, please contact the Foundation Compositio Mathematica.

This work is supported by a World Premier International Research Center Initiative (WPI initiative), MEXT, Japan, a Grant-in Aid for Scientific Research grant (No. 26287002) from the Ministry of Education, Culture, Sports, Science and Technology, Japan, and the JSPS Program for Advancing Strategic International Networks to Accelerate the Circulation of Talented Researchers. 


\section{HALL ALGEBRAS}

[AHR15]. As this fact was not explicitly mentioned in the literature, we point it out in this article. The following statement was formulated as Conjecture 1.2 in [Tod13b].

Theorem 1.1 (Ben-Bassat-Brav-Bussi-Joyce [BBBJ15], Alper-Hall-Rydh [AHR15], Theorem 2.1). Let $X$ be a smooth projective Calabi-Yau 3-fold over $\mathbb{C}$ and $\mathcal{M}$ be the moduli stack of semi-Schur objects $E \in D^{b} \operatorname{Coh}(X)$; that is, they satisfy $\operatorname{Ext}^{<0}(E, E)=0$. For $[E] \in \mathcal{M}$, let $G$ be a maximal reductive subgroup of $\operatorname{Aut}(E)$. Then there exist a $G$-invariant analytic open neighborhood $V$ of 0 in $\operatorname{Ext}^{1}(E, E)$, a $G$-invariant holomorphic function $f: V \rightarrow \mathbb{C}$ with $f(0)=\left.d f\right|_{0}=0$, and a smooth morphism of complex analytic stacks

$$
\Phi:([\{d f=0\} / G], 0) \rightarrow(\mathcal{M},[E])
$$

of relative dimension $\operatorname{dim} \operatorname{Aut}(E)-\operatorname{dim} G$.

The above result was first proved when $E \in \operatorname{Coh}(X)$ by Joyce-Song [JS12] using gauge theory. In general, by the work of Pantev-Toën-Vaquie-Vezzosi [PTVV13], the stack $\mathcal{M}$ is the truncation of a smooth derived stack with a $(-1)$-shifted symplectic structure. Using this fact, Ben-Bassat-Brav-Bussi-Joyce [BBBJ15] showed that $\mathcal{M}$ has Zariski locally an atlas which is written as a critical locus of a certain algebraic function. In particular, Theorem 1.1 was proved by them for a $\operatorname{Schur}$ object $[E] \in \mathcal{M}$; that is, $\operatorname{Hom}(E, E)=\mathbb{C}$. For a strictly semi-Schur object $[E] \in \mathcal{M}$, the remaining issue has been whether we can take the function $f$ to be invariant under the $G$-action. This issue was addressed by Bussi [Bus14], who showed a result similar to Theorem 1.1 under the assumption that $\mathcal{M}$ is Zariski locally written as a quotient stack of the form $\left[S / \mathrm{GL}_{n}(\mathbb{C})\right]$ for a quasi-projective variety $S$. It is still not known whether the last assumption holds for $\mathcal{M}$. However, the work of Alper-Hall-Rydh [AHR15] implies that locally near $[E] \in \mathcal{M}$, the stack $\mathcal{M}$ admits a smooth morphism of the form $[S / G] \rightarrow \mathcal{M}$ for an affine variety $S$ with relative $\operatorname{dimension} \operatorname{dim} \operatorname{Aut}(E)-\operatorname{dim} G$. We will see that the result of [AHR15] is enough to conclude Theorem 1.1.

Now, given Theorem 1.1, all of the Hall algebra arguments in [JS12, Bri12] apply for any heart $\mathcal{A}$ of a bounded t-structure in the derived category of coherent sheaves on a Calabi-Yau 3 -fold $X$. In particular, following [Bri12], we can construct a Poisson algebra homomorphism from the regular elements of the motivic Hall algebra of $\mathcal{A}$ to the Poisson torus, which we will review in Section 2.6. Such a statement is relevant to apply the wall-crossing formula in the derived category.

\subsection{Removing assumptions in previous papers}

The result of Theorem 1.1 was conjectured and assumed in the author's previous papers. Now we can remove that assumption from the results in [Tod10a, Theorem 1.2], [Tod13b, Theorem 1.3], [Tod11, Theorem 1.3], [Tod12, Theorem 3.11], [Tod13a, Theorem 1.5], [Tod16, Theorem 1.2] or change the statement of the Euler characteristic version to that on the honest DT invariants.

For example, let us focus on the DT/PT (where PT stands for Pandharipande-Thomas) correspondence in [Tod10a]. Let $X$ be a smooth projective Calabi-Yau 3-fold, and take

$$
\beta \in H_{2}(X, \mathbb{Z}), \quad n \in \mathbb{Z} .
$$

Associated with the above data, we have two kinds of curve-counting invariants,

$$
I_{n, \beta} \in \mathbb{Z} \text { and } P_{n, \beta} \in \mathbb{Z} \text {. }
$$

The invariant $I_{n, \beta}$ is the rank one DT invariant [MNOP06], which virtually counts subschemes $C \subset X$ with $\operatorname{dim} C \leqslant 1,[C]=\beta$, and $\chi\left(\mathcal{O}_{C}\right)=n$. On the other hand, $P_{n, \beta}$ is the PT stable pair 


\section{Y. TODA}

invariant [PT09], which virtually counts pairs $(F, s)$ where $F$ is a pure one-dimensional sheaf with $[F]=\beta$ and $\chi(F)=n$, and $s: \mathcal{O}_{X} \rightarrow F$ is a morphism which is surjective in dimension one. The above two invariants are known to be related by

$$
\sum_{n \in \mathbb{Z}} I_{n, \beta} q^{n}=M(-q)^{e(X)} \cdot \sum_{n \in \mathbb{Z}} P_{n, \beta} q^{n} .
$$

Here, $M(q)$ is the MacMahon function

$$
M(q)=\prod_{k \geqslant 1}\left(1-q^{k}\right)^{-k}
$$

The formula (1.1) was conjectured in [PT09]; its Euler characteristic version was proved in [Tod10a, ST11], and the Behrend function-weighted version was finally proved by Bridgeland [Bri11]. As we remarked in the appendix of an earlier version (arXiv:0902.4371) of [Tod10a], the argument of [Tod10a] also proves the formula (1.1) if we know Theorem 1.1. So we can now prove formula (1.1) using the argument of [Tod10a] without any assumption.

In any case, formula (1.1) was proved by Bridgeland [Bri11] without using Theorem 1.1, so Theorem 1.1 is not essential in proving (1.1). On the other hand, it seems that Theorem 1.1 is essential in proving the higher-rank version of formula (1.1), which we discuss in the next subsection. Before this, let us discuss the difference between the arguments in [Tod10a] and [Bri11]. In [Tod10a], we regarded a subscheme $C \subset X$ as an ideal sheaf $I_{C}$ and a stable pair as a twoterm complex $\left(\mathcal{O}_{X} \stackrel{s}{\rightarrow} F\right)$. As they are rank one objects in the derived category, we used the Hall algebra of the heart of some t-structure to show the Euler characteristic version of (1.1). On the other hand, in [Bri11], a subscheme and a stable pair were regarded as coherent systems, which are one-dimensional sheaves together with sections. The latter viewpoint has an advantage in the fact that everything can be worked out in the Hall algebra of one-dimensional sheaves. So the result of Theorem 1.1 for coherent sheaves, which was already shown in [JS12], was enough to prove formula (1.1).

However, the above interpretation of a sheaf as a coherent system is only possible for a rank one object. A higher-rank stable sheaf is not necessarily regarded as data $W \otimes \mathcal{O}_{X} \rightarrow F$ for some finite-dimensional vector space $W$ and a one-dimensional sheaf $F$. So it is not obvious how to study the higher-rank DT invariants using Hall algebras of one-dimensional sheaves as in [Bri11].

\subsection{Wall-crossing formula for higher-rank objects}

The second purpose of this article is to study higher-rank DT invariants, for example giving a higher-rank analogue of formula (1.1). Here, we emphasize that in contrast with the rank one case, Theorem 1.1 is essential to give a rigorous proof. Let us take an ample divisor $\omega$ on $X$ and an element

$$
(r, D,-\beta,-n) \in H^{0}(X) \oplus H^{2}(X) \oplus H^{4}(X) \oplus H^{6}(X)
$$

such that $r \geqslant 1$ and $\left(r, D \cdot \omega^{2}\right)$ are coprime. Let

$$
\operatorname{DT}(r, D,-\beta,-n) \in \mathbb{Z}
$$

be the DT invariant which virtually counts $\omega$-slope stable sheaves $E \in \operatorname{Coh}(X)$ whose Chern character coincides with (1.2).

We define a PT stable object as an object

$$
I^{\bullet} \in D^{b} \operatorname{Coh}(X)
$$




\section{HALL ALGEBRAS}

such that $\mathcal{H}^{i}\left(I^{\bullet}\right)=0$ for $i \neq 0,1, \mathcal{H}^{0}\left(I^{\bullet}\right)$ is a $\omega$-slope stable sheaf, and $\mathcal{H}^{1}\left(I^{\bullet}\right)$ is zero-dimensional. This notion appeared in Lo's work [Lo12] in describing certain polynomial-stable objects [Bay09]. A PT stable object is a PT stable pair in the rank one case, but it is not necessarily written as a reasonable pair in a higher-rank case (cf. Section 3.2). We can define the invariant

$$
\operatorname{PT}(r, D,-\beta,-n) \in \mathbb{Z},
$$

which virtually counts objects (1.4) whose Chern character is $(1.2)$. When $(r, D)=(1,0)$, the invariants (1.3) and (1.5) coincide with $I_{n, \beta}$ and $P_{n, \beta}$, respectively. The following is a higher-rank analogue of formula (1.1).

Theorem 1.2 (Theorem 3.18). For a fixed $(r, D, \beta)$, we have the following formula:

$$
\sum_{6 n \in \mathbb{Z}} \mathrm{DT}(r, D,-\beta,-n) q^{n}=M\left((-1)^{r} q\right)^{r \cdot e(X)} \sum_{6 n \in \mathbb{Z}} \mathrm{PT}(r, D,-\beta,-n) q^{n} .
$$

We note that formula (1.1) is the special case of $(1.6)$ for $(r, D)=(1,0)$. Indeed, in the rank one case, the proof is essentially the same as in [Tod10a], where we studied the wall-crossing phenomena in the category generated by $\mathcal{O}_{X}$ and one-dimensional sheaves shifted by $[-1]$ in the derived category. We will also construct a similar abelian category and investigate a wall-crossing phenomenon. However, in this article we simplified several arguments by considering certain nested torsion pairs in the abelian category rather than studying the weak-stability conditions. Also, as we work with the Hall algebra of a t-structure in the derived category (rather than that of one-dimensional sheaves as in [Bri11]), the use of Theorem 1.1 is essential for the proof.

The generating series of PT invariants was also conjectured to be a rational function in [PT09], which is required to formulate the $\mathrm{PT} / \mathrm{GW}$ correspondence (where $\mathrm{GW}$ stands for GromovWitten). The rationality conjecture was proved for the Euler characteristic version in [Tod10b] and finally proved by Bridgeland [Bri11]. In the higher-rank case, we have the following similar rationality statement.

Theorem 1.3 (Corollary 4.9). For a fixed $(r, D, \beta)$, we can write

$$
\sum_{6 n \in \mathbb{Z}} \operatorname{PT}(r, D,-\beta,-n) q^{n}=F(q) \cdot G\left(q^{1 / 6}\right)
$$

where $F(q)$ is the Laurent expansion of a rational function of $q$ and $G\left(q^{1 / 6}\right)$ is a Laurent polynomial in $q^{1 / 6}$ with integer coefficients.

Again, the proof is essentially the same as in [Tod10b], but the proof is much simplified, and we use Theorem 1.1 for the rigorous proof. Also, unlike in the rank one case, the rational functions $F(q)$ and $G\left(q^{1 / 6}\right)$ are not necessarily invariant under $q \leftrightarrow q^{-1}$ and $q^{1 / 6} \leftrightarrow q^{-1 / 6}$, respectively, in the higher-rank case. By combining Theorems 1.2 and 1.3, we obtain the formula

$$
\sum_{6 n \in \mathbb{Z}} \mathrm{DT}(r, D,-\beta,-n) q^{n}=M\left((-1)^{r} q\right)^{r \cdot e(X)} \cdot F(q) \cdot G\left(q^{1 / 6}\right) .
$$

This formula is a new structure result on DT invariants which can be applied for any positive rank.

\subsection{Related works}

A result similar to Theorem 1.1 was once announced by Behrend-Getzler. Recently, Jiang [Jia18] proved the Behrend function identities given in Theorem 2.7 using the cyclic $L_{\infty}$-algebra technique and unpublished work by Behrend-Getzler. 


\section{Y. TODA}

So far, there exist some articles in which a higher-rank analogue of DT theory or PT theory has been studied [Tod10c, Sto12, Nag10, CDP10, She11]. In these articles, all the higher-rank objects are of the form $\left(W \otimes \mathcal{O}_{X} \rightarrow F\right)$; this does not cover all of the stable sheaves as we already mentioned. So our situation is much more general than that in the above articles.

It is a natural problem to extend the results of Theorems 1.2 and 1.3 to the motivic DT invariants introduced by Kontsevich-Soibelman [KS08]. Still, there exist some technical issues in this extension, for example the existence of an orientation data, but the number of issues is decreasing due to the recent progress on the rigorous foundation of motivic DT theory (cf. [BJM19, Lê15, DM15, Jia16]).

\section{Hall algebras in the derived category}

\section{$2.1 d$-critical stacks}

We first recall Joyce's notion of $d$-critical stacks introduced in [Joy15]. For any algebraic stack $\mathcal{X}$, Joyce constructed a sheaf of vector spaces $\mathcal{S}_{\mathcal{X}}^{0}$ satisfying the following property. For any scheme $V$, a smooth morphism $V \rightarrow \mathcal{X}$, and a closed embedding $i: V \hookrightarrow U$ for a smooth scheme $U$, there is an exact sequence

$$
\left.0 \rightarrow \mathcal{S}_{\mathcal{X}}^{0}\right|_{V} \oplus \mathbb{C}_{V} \rightarrow i^{-1} \mathcal{O}_{U} / I^{2} \stackrel{d}{\rightarrow} i^{-1} \Omega_{U} / I \cdot i^{-1} \Omega_{U}
$$

Here $I \subset i^{-1} \mathcal{O}_{U}$ is the ideal sheaf of functions vanishing on $V$, and $\mathbb{C}_{V}$ is the constant sheaf on $V$. For example, if there is a regular function $f: U \rightarrow \mathbb{A}^{1}$ with $V=\{d f=0\}$ and $\left.f\right|_{V^{\text {red }}}=0$, then

$$
s=f+I^{2} \in \Gamma\left(V,\left.\mathcal{S}_{\mathcal{X}}^{0}\right|_{V}\right) .
$$

By definition, a pair $(\mathcal{X}, s)$ for an algebraic stack $\mathcal{X}$ and $s \in H^{0}\left(\mathcal{S}_{\mathcal{X}}^{0}\right)$ is called a $d$-critical stack if for any scheme $V$ and a smooth morphism $V \rightarrow \mathcal{X}$, the section $\left.s\right|_{V} \in \Gamma\left(V,\left.\mathcal{S}_{\mathcal{X}}^{0}\right|_{V}\right)$ can be written as $(2.1)$ for some data $(U, f, i)$. In this case, the data

$$
(V, U, f, i)
$$

is called a $d$-critical chart. Roughly speaking, a $d$-critical stack is an algebraic stack which locally has an atlas given by the critical locus of some function $f$, and the section $s$ remembers the function $f$.

\subsection{Luna étale slice theorem for algebraic stacks}

It is well known that the stack of coherent sheaves on a projective scheme can be Zariski locally written as a quotient stack. However, such a result is not known for the stack of objects in the derived category of coherent sheaves. The following result by Alper-Hall-Rydh [AHR15], simplified in the $k=\mathbb{C}$ case, will be useful to settle the above issue.

ThEOREM 2.1 ([AHR15, Theorem 1.2]). Let $\mathcal{X}$ be a quasi-separated algebraic stack, locally of finite type over $\mathbb{C}$ with affine geometric stabilizers. Let $x \in \mathcal{X}$ be a point and $G \subset \operatorname{Aut}(x)$ a reductive subgroup scheme. Then there exist an affine scheme $S$ with a $G$-action, a point $p \in S$ fixed by $G$, and a smooth morphism

$$
\Phi:([S / G], p) \rightarrow(\mathcal{X}, x) .
$$

We can say more on the above result, which we mention in the following remark. 


\section{HALL ALGEBRAS}

Remark 2.2. From the construction of $S$ in the proof of [AHR15, Theorem 1.2], we have the 2-Cartesian diagrams

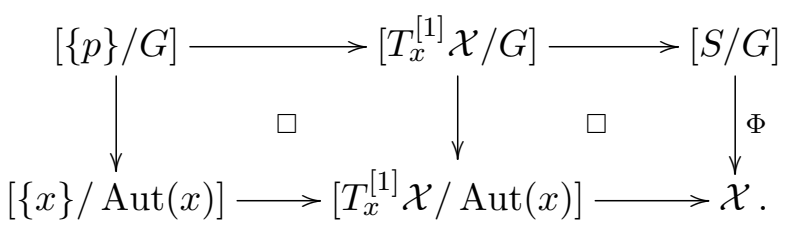

Here $T_{x}^{[1]} \mathcal{X} \subset T_{x} \mathcal{X}$ is the first-order infinitesimal neighborhood at zero, the top horizontal morphisms are induced by a $G$-equivariant embedding $T_{x}^{[1]} \mathcal{X} \hookrightarrow S$ sending 0 to $p$, the bottomleft morphism sends $\{x\}$ to 0 , and the bottom-right morphism is induced by the natural map $T_{x}^{[1]} \mathcal{X} \rightarrow \mathcal{X}$. In particular, the morphism $\Phi$ is of relative $\operatorname{dimension} \operatorname{dim} \operatorname{Aut}(x)-\operatorname{dim} G$, the morphism $G=\operatorname{Aut}(p) \rightarrow \operatorname{Aut}(x)$ between stabilizers induced by $\Phi$ coincides with the inclusion $G \subset \operatorname{Aut}(x)$, and the induced map on tangent spaces

$$
\left.d \Phi\right|_{p}: T_{p}[S / G] \rightarrow T_{x} \mathcal{X}
$$

is an isomorphism.

\subsection{Proof of Theorem 1.1}

In what follows, $X$ is a smooth projective Calabi-Yau 3 -fold over $\mathbb{C}$; that is, $K_{X}=0$ and $H^{1}\left(X, \mathcal{O}_{X}\right)=0$. We denote by $\mathcal{M}$ the stack of objects

$$
E \in D^{b} \operatorname{Coh}(X), \quad \operatorname{Ext}^{<0}(E, E)=0 .
$$

By a result of Lieblich [Lie06], the stack $\mathcal{M}$ is an algebraic stack that is locally of finite type. Using the theory of Joyce's $d$-critical stacks and Theorem 2.1, one can show an algebraic version of Theorem 1.1. The following Theorem 2.3 is a generalization of [Bus14, Theorem 4.3] and obviously implies Theorem 1.1.

Theorem 2.3. For $[E] \in \mathcal{M}$, let $G \subset \operatorname{Aut}(E)$ be the maximal reductive subgroup. Then there exist a smooth affine scheme $U$ with a $G$-action, a $G$-invariant point $p \in U$, a $G$-invariant regular function $f: U \rightarrow \mathbb{A}^{1}$ with $f(p)=\left.d f\right|_{p}=0$, and a smooth morphism

$$
\Phi:([\{d f=0\} / G], p) \rightarrow(\mathcal{M},[E])
$$

with relative dimension $\operatorname{dim} \operatorname{Aut}(E)-\operatorname{dim} G$. Moreover, let $g \in G$ act on $\operatorname{Ext}^{1}(E, E)$ by $\epsilon \mapsto$ $g \circ \epsilon \circ g^{-1}$. Then there is a $G$-equivariant étale morphism $u: U \rightarrow \operatorname{Ext}^{1}(E, E)$ with $u(p)=0$, and under the natural identification $T_{[E]} \mathcal{M}=\operatorname{Ext}^{1}(E, E)$, we have

$$
\left.d \Phi\right|_{p}=\left.d u\right|_{p}: T_{p} U \rightarrow T_{[E]} \mathcal{M} .
$$

Proof. By [Lie06], the stack $\mathcal{M}$ is locally quasi-separated. The geometric stabilizer at $[E] \in \mathcal{M}$ is $\operatorname{Aut}(E)$, which is an affine algebraic group as it is an open subscheme of $\operatorname{Hom}(E, E)$. Therefore, applying Theorem 2.1 and keeping in mind Remark 2.2, we see that there exist an affine scheme $S$ with a $G$-action, a $G$-invariant point $p \in S$, and a smooth morphism

$$
\Phi:[S / G] \rightarrow \mathcal{M}
$$

which sends $p$ to $[E]$ with relative $\operatorname{dimension} \operatorname{dim} \operatorname{Aut}(E)-\operatorname{dim} G$.

By [BBBJ15, Corollary 3.19], the stack $\mathcal{M}$ extends to the $d$-critical stack $(\mathcal{M}, s)$. This fact is based on the result by Pantev-Töen-Vaquie-Vezzosi [PTVV13] that the stack $\mathcal{M}$ is the truncation of a smooth derived stack with a $(-1)$-shifted symplectic structure. Since (2.3) is a smooth 


\section{Y. TODA}

morphism, by [Joy15, Proposition 2.8], the pull-back

$$
\Phi^{*} s \in \Gamma\left([S / G], \mathcal{S}_{[S / G]}^{0}\right)
$$

gives the $d$-critical stack $\left([S / G], \Phi^{*} s\right)$. But by [Joy15, Example 2.55], a $d$-critical structure on the quotient stack $[S / G]$ is equivalent to a $G$-invariant $d$-critical structure on $S$ defined in [Joy 15 , Definition 2.40]. Since $G$ is reductive, $S$ is affine, and $p \in S$ is fixed by $G$, we can apply [Joy 15 , Proposition 2.43, Remark 2.47] to conclude the following: after shrinking $S$ in a neighborhood of $p \in S$ if necessary, there exists a $G$-invariant critical chart $(S, U, f, i)$ such that $\operatorname{dim} U=\operatorname{dim} T_{p} S$. Here by the definition of a $G$-invariant $d$-critical chart in [Joy15, Definition 2.40], the object $U$ is a smooth scheme with a $G$-action, $f: U \rightarrow \mathbb{A}^{1}$ is a $G$-invariant function, and $i: S \hookrightarrow U$ is a $G$-equivariant embedding such that $S=\{d f=0\}$ and $\left.f\right|_{S^{\text {red }}}=0$. Therefore, the morphism (2.3) gives a smooth morphism (2.2) as desired.

By Remark 2.2, the morphism $\left.d \Phi\right|_{p}: T_{p}[S / G] \rightarrow T_{[E]} \mathcal{M}$ is an isomorphism. Since we have

$$
T_{p}[S / G]=T_{p} S=T_{p} U, \quad T_{[E]} \mathcal{M}=\operatorname{Ext}^{1}(E, E)
$$

and the embedding $i$ is $G$-equivariant, the morphism $\left.d \Phi\right|_{p}$ induces the $G$-equivariant isomorphism $T_{p} U \stackrel{\cong}{\rightrightarrows} \operatorname{Ext}^{1}(E, E)$. Therefore, by shrinking $(S, U)$ if necessary, we can construct a desired $G$ equivariant étale morphism $u: U \rightarrow \operatorname{Ext}^{1}(E, E)$.

Remark 2.4. The result of Theorem 2.3 also holds when $X$ is a some non-compact Calabi-Yau 3fold and $E \in D^{b} \operatorname{Coh}(X)$ has compact support satisfying $\operatorname{Ext}^{<0}(E, E)=0$. The only obstruction is the existence of a $(-1)$-shifted symplectic structure on the derived enhancement of the moduli stack of compactly supported objects. As far as the author knows, this is only known when $X=Z \backslash Y$ for a smooth projective 4 -fold $Z$ and $Y \in\left|-K_{Z}\right|$ (see [Bus14, Theorem 5.2]).

\subsection{Behrend function identities}

In [Beh09], Behrend constructed a canonical constructible function on any scheme, called the Behrend function. The Behrend function is naturally extended to algebraic stacks [JS12]. Using Theorem 1.1, the Behrend function $\nu_{\mathcal{M}}: \mathcal{M} \rightarrow \mathbb{Z}$ on the moduli stack $\mathcal{M}$ in Section 2.3 is described as follows:

$$
\nu_{\mathcal{M}}([E])=(-1)^{\operatorname{hom}(E, E)-\operatorname{ext}^{1}(E, E)}\left(1-e\left(M_{f}(0)\right)\right),
$$

where $M_{f}(0)$ is the Milnor fiber of the function $f: V \rightarrow \mathbb{C}$ at $0 \in V$ in Theorem 1.1 and $e(-)$ is the topological Euler number. The result of Theorem 1.1 implies the analogue of the Behrend function identities proved for coherent sheaves in [JS12].

We introduce some notation. First, for a constructible function $\nu$ on a scheme $M$, we set

$$
\int_{M} \nu d e:=\sum_{m \in \mathbb{Z}} m \cdot e\left(\nu^{-1}(m)\right) .
$$

Next, for $E_{1}, E_{2} \in D^{b} \operatorname{Coh}(X)$, their Euler pairing is defined by

$$
\chi\left(E_{1}, E_{2}\right):=\sum_{i \in \mathbb{Z}}(-1)^{i} \operatorname{ext}^{i}\left(E_{1}, E_{2}\right) .
$$

Below, we fix the heart of a bounded t-structure $\mathcal{A} \subset D^{b} \operatorname{Coh}(X)$ on $D^{b} \operatorname{Coh}(X)$.

Remark 2.5. Note that any object $E \in \mathcal{A}$ satisfies $\operatorname{Ext}^{<0}(E, E)=0$ by the definition of the t-structure, hence determines the point $[E] \in \mathcal{M}$. 


\section{HALL ALGEBRAS}

We have the following lemma.

Lemma 2.6. For $E_{1}, E_{2} \in \mathcal{A}$, we have

$$
\chi\left(E_{1}, E_{2}\right)=\operatorname{hom}\left(E_{1}, E_{2}\right)-\operatorname{ext}^{1}\left(E_{1}, E_{2}\right)+\operatorname{ext}^{1}\left(E_{2}, E_{1}\right)-\operatorname{hom}\left(E_{2}, E_{1}\right) .
$$

Proof. The lemma follows from $\operatorname{ext}^{i}\left(E_{1}, E_{2}\right)=0$ for $i<0$ by the definition of the t-structure and the identity $\operatorname{ext}^{i}\left(E_{1}, E_{2}\right)=\operatorname{ext}^{3-i}\left(E_{2}, E_{1}\right)$ from Serre duality.

Third, for $E_{1}, E_{2} \in \mathcal{A}$ and $\xi \in \mathbb{P}\left(\operatorname{Ext}^{1}\left(E_{1}, E_{2}\right)\right)$, we denote by $E_{\xi} \in \mathcal{A}$ the object given by the extension class corresponding to $\xi$ :

$$
0 \rightarrow E_{2} \rightarrow E_{\xi} \rightarrow E_{1} \rightarrow 0
$$

After the above preparation, we can state the generalization of [JS12, Theorem 5.11] as follows.

Theorem 2.7. For any heart of a bounded t-structure $\mathcal{A} \subset D^{b} \operatorname{Coh}(X)$ and $E_{1}, E_{2} \in \mathcal{A}$, we have the following identities:

$$
\begin{aligned}
\nu_{\mathcal{M}}\left(\left[E_{1} \oplus E_{2}\right]\right) & =(-1)^{\chi\left(E_{1}, E_{2}\right)} \nu_{\mathcal{M}}\left(\left[E_{1}\right]\right) \nu_{\mathcal{M}}\left(\left[E_{2}\right]\right) \\
\int_{\xi \in \mathbb{P}\left(\operatorname{Ext}^{1}\left(E_{2}, E_{1}\right)\right)} \nu_{\mathcal{M}}\left(E_{\xi}\right) d e & -\int_{\xi \in \mathbb{P}\left(\operatorname{Ext}^{1}\left(E_{1}, E_{2}\right)\right)} \nu_{\mathcal{M}}\left(E_{\xi}\right) d e \\
& =\left(\operatorname{ext}^{1}\left(E_{2}, E_{1}\right)-\operatorname{ext}^{1}\left(E_{1}, E_{2}\right)\right) \nu_{\mathcal{M}}\left(\left[E_{1} \oplus E_{2}\right]\right) .
\end{aligned}
$$

Proof. The result is proved for $\mathcal{A}=\operatorname{Coh}(X)$ in [JS12, Theorem 5.11]. For a general $\mathcal{A}$, the result follows from the same argument of [JS12, Theorem 5.11], using Theorem 1.1 instead of [JS12, Theorem 5.5] and keeping in mind Lemma 2.6.

Remark 2.8. By the proof of Theorem 1.1, the weaker statement of Theorem 1.1 holds after replacing $G$ with its maximal torus. As proved in [Bus14, Theorem 4.2], the latter weaker version is enough to prove Theorem 2.7.

\subsection{Hall algebras}

We recall the notion of motivic Hall algebras following [Bri12]. Let $\mathcal{S}$ be an algebraic stack locally of finite type with affine geometric stabilizers. By definition, $K(\mathrm{St} / \mathcal{S})$ is defined to be the $\mathbb{Q}$-vector space generated by isomorphism classes of symbols

$$
[\mathcal{X} \stackrel{\rho}{\rightarrow} \mathcal{S}]
$$

where $\mathcal{X}$ is an algebraic stack of finite type with affine geometric stabilizers. The relations are generated by the following (cf. [Bri12, Definition 3.10]):

(1) For every pair of $\mathcal{X}_{1}, \mathcal{X}_{2}$, we have

$$
\left[\mathcal{X}_{1} \sqcup \mathcal{X}_{2} \stackrel{\rho_{1} \sqcup \rho_{2}}{\longrightarrow} \mathcal{S}\right]=\left[\mathcal{X}_{1} \stackrel{\rho_{1}}{\longrightarrow} \mathcal{S}\right]+\left[\mathcal{X}_{2} \stackrel{\rho_{2}}{\longrightarrow} \mathcal{S}\right]
$$

(2) For every geometric bijection $\rho: \mathcal{X}_{1} \rightarrow \mathcal{X}_{2}$ and morphism $\rho^{\prime}: \mathcal{X}_{2} \rightarrow \mathcal{S}$, we have

$$
\left[\mathcal{X}_{1} \stackrel{\rho \circ \rho^{\prime}}{\rightarrow} \mathcal{S}\right]=\left[\mathcal{X}_{2} \stackrel{\rho^{\prime}}{\rightarrow} \mathcal{S}\right]
$$

(3) For every pair of Zariski locally trivial fibrations $h_{i}: \mathcal{X}_{i} \rightarrow \mathcal{Y}$ with the same fibers and every morphism $g: \mathcal{Y} \rightarrow \mathcal{S}$, we have

$$
\left[\mathcal{X}_{1} \stackrel{g \circ h_{1}}{\longrightarrow} \mathcal{S}\right]=\left[\mathcal{X}_{2} \stackrel{g \circ h_{2}}{\longrightarrow} \mathcal{S}\right]
$$




\section{Y. TODA}

Let $\mathcal{A} \subset D^{b} \operatorname{Coh}(X)$ be the heart of a bounded t-structure such that the substack

$$
\mathcal{O} b j(\mathcal{A}) \subset \mathcal{M}
$$

consisting of objects in $\mathcal{A}$ is an open substack of $\mathcal{M}$. Then the motivic Hall algebra of $\mathcal{A}$ is defined as

$$
H(\mathcal{A}):=K(\operatorname{St} / \mathcal{O} b j(\mathcal{A})) .
$$

Set $K(\mathrm{St} / \mathbb{C}):=K(\mathrm{St} / \mathrm{Spec} \mathbb{C})$. Note that $H(\mathcal{A})$ is naturally a $K(\mathrm{St} / \mathbb{C})$-module via

$$
[\mathcal{X} \rightarrow \operatorname{Spec} \mathbb{C}] \cdot[\mathcal{Y} \stackrel{\rho}{\rightarrow} \mathcal{O} b j(\mathcal{A})]=[\mathcal{X} \times \mathcal{Y} \stackrel{p}{\rightarrow} \mathcal{Y} \stackrel{\rho}{\rightarrow} \mathcal{O} b j(\mathcal{A})]
$$

where $p$ is the projection. There is an associative $K(\mathrm{St} / \mathbb{C})$-algebra structure $*$ on $H(\mathcal{A})$ based on the Ringel-Hall algebras. Let $\mathcal{E} x(\mathcal{A})$ be the stack of short exact sequences

$$
0 \rightarrow E_{1} \rightarrow E_{3} \rightarrow E_{2} \rightarrow 0
$$

in $\mathcal{A}$ and $p_{i}: \mathcal{E} x(\mathcal{A}) \rightarrow \mathcal{O} b j(\mathcal{A})$ the 1 -morphism sending $E_{\bullet}$ to $E_{i}$. The stack $\mathcal{E} x(\mathcal{A})$ is an algebraic stack that is locally of finite type [BCR18, Appendix B]. The $*$-product on $H(\mathcal{A})$ is given by

$$
\left[\mathcal{X}_{1} \stackrel{\rho_{1}}{\longrightarrow} \mathcal{O} b j(\mathcal{A})\right] *\left[\mathcal{X}_{2} \stackrel{\rho_{2}}{\longrightarrow} \mathcal{O} b j(\mathcal{A})\right]=\left[\mathcal{X}_{3} \stackrel{\rho_{3}}{\longrightarrow} \mathcal{O} b j(\mathcal{A})\right]
$$

where $\left(\mathcal{X}_{3}, \rho_{3}=p_{3} \circ\left(\rho_{1}^{\prime}, \rho_{2}^{\prime}\right)\right)$ is given by the Cartesian diagram

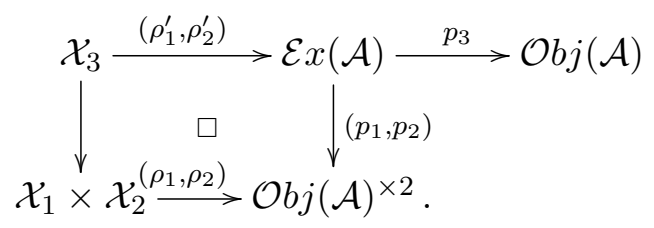

The unit is given by $1=[\operatorname{Spec} \mathbb{C} \rightarrow \mathcal{O} b j(\mathcal{A})]$, which corresponds to $0 \in \mathcal{A}$.

Let $\Gamma$ be the image of the Chern character map, $\Gamma:=\operatorname{Im}\left(\operatorname{ch}: K(X) \rightarrow H^{*}(X, \mathbb{Q})\right)$. Then $\Gamma$ is a finitely generated free abelian group. The stack (2.6) decomposes into the disjoint union of open and closed substacks

$$
\mathcal{O} b j(\mathcal{A})=\coprod_{v \in \Gamma} \mathcal{O} b j_{v}(\mathcal{A})
$$

where $\mathcal{O} b j_{v}(\mathcal{A})$ is the stack of objects in $\mathcal{A}$ with Chern character $v$. The algebra $H(\mathcal{A})$ is $\Gamma$ graded, $H(\mathcal{A})=\bigoplus_{v \in \Gamma} H_{v}(\mathcal{A})$, where $H_{v}(\mathcal{A})$ is spanned by $[\mathcal{X} \rightarrow \mathcal{O} b j(\mathcal{A})]$, which factors through $\mathcal{O} b j_{v}(\mathcal{A}) \subset \mathcal{O b j}(\mathcal{A})$.

\subsection{Poisson algebra homomorphism}

It is easy to see that the affine line $\mathbb{L}:=\left[\mathbb{A}^{1} \rightarrow \mathrm{Spec} \mathbb{C}\right] \in K(\mathrm{St} / \mathbb{C})$ is an invertible element. We define the subalgebra $K(\operatorname{Var} / \mathbb{C})\left[\mathbb{L}^{-1}\right] \subset K(\mathrm{St} / \mathbb{C})$ to be generated by $\mathbb{L}^{-1}$ and $[Y \rightarrow$ Spec $\mathbb{C}]$ for a variety $Y$. The $K(\operatorname{Var} / \mathbb{C})\left[\mathbb{L}^{-1}\right]$-submodule

$$
H^{\mathrm{reg}}(\mathcal{A}) \subset H(\mathcal{A})
$$

is defined to be spanned by $[Z \rightarrow \mathcal{O} b j(\mathcal{A})]$, where $Z$ is a variety. An element of $H^{\text {reg }}(\mathcal{A})$ is called regular. By [Bri12, Theorem 5.1], the submodule (2.8) is indeed a subalgebra with respect to the *-product. Moreover, the quotient

$$
H^{\mathrm{sc}}(\mathcal{A}):=H^{\mathrm{reg}}(\mathcal{A}) /(\mathbb{L}-1) H^{\mathrm{reg}}(\mathcal{A})
$$


is a commutative algebra. Therefore, for $f, g \in H^{\mathrm{sc}}(\mathcal{A})$, we can define the following bracket on $H^{\mathrm{sc}}(\mathcal{A})$ :

$$
\{f, g\}:=\frac{f * g-g * f}{\mathbb{L}-1} .
$$

By the $*$-product together with the above bracket $\{-,-\}$, we have a Poisson algebra structure on $H^{\mathrm{sc}}(\mathcal{A})$.

We define another Poisson algebra $C(X)$ to be

$$
C(X):=\bigoplus_{v \in \Gamma} \mathbb{Q} \cdot c_{v} .
$$

Note that by the Riemann-Roch theorem, the Euler pairing (2.4) descends to the anti-symmetric bilinear form $\chi: \Gamma \times \Gamma \rightarrow \Gamma$. We will only use the following computation, which is an easy consequence of the Riemann-Roch theorem:

$$
\chi\left(\left(0,0,-\beta_{1},-n_{1}\right),\left(r, D,-\beta_{2},-n_{2}\right)\right)=r n_{1}-D \beta_{1} .
$$

Here and in what follows, we use the notation (1.2) for the elements in $\Gamma$. The *-product on $C(X)$ is defined by $c_{v_{1}} * c_{v_{2}}=(-1)^{\chi\left(v_{1}, v_{2}\right)} c_{v_{1}+v_{2}}$. The Poisson bracket on $C(X)$ is defined by $\left\{c_{v_{1}}, c_{v_{2}}\right\}=$ $(-1)^{\chi\left(v_{1}, v_{2}\right)} \chi\left(v_{1}, v_{2}\right) c_{v_{1}+v_{2}}$. The result of Theorem 1.1 leads to the following result.

Theorem 2.9 ([Bri12, Theorem 5.2]). There is a Poisson algebra homomorphism

$$
I: H^{\mathrm{sc}}(\mathcal{A}) \rightarrow C(X)
$$

such that for a variety $Z$ with a morphism $\rho: Z \rightarrow \mathcal{O} b j(\mathcal{A})$ which factors through $\mathcal{O} b j_{v}(\mathcal{A})$, we have

$$
I([Z \stackrel{\rho}{\rightarrow} \mathcal{O} b j(\mathcal{A})])=\left(\int_{Z} \rho^{*} \nu_{\mathcal{M}}\right) \cdot c_{v} .
$$

Here $\nu_{\mathcal{M}}$ is the Behrend function on $\mathcal{M}$ restricted to $\mathcal{O b j}(\mathcal{A})$.

Proof. By Theorem 2.7, the assumption in [Bri12, Theorem 5.2] is satisfied; hence the result holds.

\section{Higher-rank DT/PT correspondence}

In this section, we prove Theorem 1.2.

\subsection{Donaldson-Thomas invariants}

Below, we fix an ample divisor $\omega$ on a Calabi-Yau 3-fold $X$. Let us take an element

$$
v=(r, D,-\beta,-n) \in \Gamma
$$

in the notation (1.2). Since $\Gamma$ is the image of the Chern character map, we may assume $r \in \mathbb{Z}$, $D \in H^{2}(X, \mathbb{Z}), 2 \beta \in H^{4}(X, \mathbb{Z})$, and $6 n \in \mathbb{Z}$. We also assume

$$
r \in \mathbb{Z}_{\geqslant 1} \text { and } \operatorname{gcd}\left(r, D \cdot \omega^{2}\right)=1 .
$$

Recall that for a coherent sheaf $E$ on $X$, its slope function $\mu_{\omega}$ is defined by

$$
\mu_{\omega}(E):=\frac{c_{1}(E) \cdot \omega^{2}}{\operatorname{rank}(E)} \in \mathbb{Q} \cup\{\infty\}
$$




\section{Y. TODA}

A sheaf $E$ is called $\mu_{\omega}$-semistable if for any non-trivial subsheaf $F \subset E$, we have $\mu_{\omega}(F) \leqslant$ $\mu_{\omega}(E / F)$. It is called $\mu_{\omega}$ stable if the inequality is strict. By the coprime condition (3.2), the moduli space $M_{\mathrm{DT}}(r, D,-\beta,-n)$ of $\mu_{\omega}$-semistable sheaves with Chern character $(r, D,-\beta,-n)$ consists of $\mu_{\omega}$-stable sheaves; moreover, it is a projective scheme [HL97]. Also by the CY3 condition on $X$, the space $M_{\mathrm{DT}}(r, D,-\beta,-n)$ is equipped with a symmetric perfect obstruction theory and the associated zero-dimensional virtual class [Tho00, BF08]. The Donaldson-Thomas (DT) invariant is defined by

$$
\mathrm{DT}(r, D,-\beta,-n):=\int_{\left[M_{\mathrm{DT}}(r, D,-\beta,-n)\right]^{\mathrm{vir}}} 1 .
$$

By fixing $(r, D)$, we define the generating series

$$
\operatorname{DT}_{r, D}(q, t):=\sum_{\beta, n} \operatorname{DT}(r, D,-\beta,-n) q^{n} t^{\beta} .
$$

\subsection{Higher-rank Pandharipande-Thomas theory}

We introduce the higher-rank version of Pandharipande-Thomas (PT) theory. The following notion is found in Lo's work [Lo12] in the analysis of Bayer's polynomial stability conditions [Bay09].

Definition 3.1. An object $I^{\bullet} \in D^{b} \operatorname{Coh}(X)$ is called PT-(semi)stable if the following conditions are satisfied:

(i) We have $\mathcal{H}^{i}\left(I^{\bullet}\right)=0$ for $i \neq 0,1$.

(ii) The object $\mathcal{H}^{0}\left(I^{\bullet}\right)$ is $\mu_{\omega^{-}}$(semi)stable and $\mathcal{H}^{1}\left(I^{\bullet}\right)$ is zero-dimensional.

(iii) We have $\operatorname{Hom}\left(Q[-1], I^{\bullet}\right)=0$ for any zero-dimensional sheaf $Q$.

Let us consider the relationship of this notion to the rank one PT theory in [PT09].

Example 3.2. Let $\mathcal{E}$ be a locally free $\mu_{\omega}$-(semi)stable sheaf on $X, F$ a pure one-dimensional sheaf on $X$, and $s: \mathcal{E} \rightarrow F$ a morphism which is surjective in dimension one. Then the object

$$
I^{\bullet}=(\mathcal{E} \stackrel{s}{\rightarrow} F) \in D^{b} \operatorname{Coh}(X)
$$

with $\mathcal{E}$ located in degree zero is a PT (semi)stable object. If $\mathcal{E}=\mathcal{O}_{X}$, then it is nothing but the stable pair in [PT09].

If an object $I^{\bullet} \in D^{b} \operatorname{Coh}(X)$ is of rank one, then it is proved in [Tod10a, Lemma 3.11] that $I^{\bullet}$ is PT semistable if and only if it is a two-term complex (3.5) with $\mathcal{E}$ a line bundle. The same argument easily shows the following.

Lemma 3.3. A PT semistable object $I^{\bullet} \in D^{b} \operatorname{Coh}(X)$ with $\operatorname{rank}\left(I^{\bullet}\right)>0$ is quasi-isomorphic to a two-term complex of the form (3.5) if and only if $\mathcal{H}^{0}\left(I^{\bullet}\right)^{\vee \vee}$ is locally free.

If the rank is bigger than one, a reflexive sheaf may not be locally free. The following example gives an exotic PT stable object.

Example 3.4. Let $\mathcal{U}$ be a $\mu_{\omega}$-stable reflexive sheaf which is not locally free at $x \in X$. Then $\operatorname{Ext}^{2}\left(\mathcal{O}_{x}, U\right)=\operatorname{Ext}^{1}\left(U, \mathcal{O}_{x}\right)^{\vee} \neq 0$. The object $I^{\bullet}$ given by a non-trivial extension

$$
\mathcal{U} \rightarrow I^{\bullet} \rightarrow \mathcal{O}_{x}[-1]
$$

is a PT stable object. However, as we will see below, $I^{\bullet}$ is not a two-term complex similar to the usual stable pair in the rank one case. 


\section{HALL ALGEBRAS}

LEMma 3.5. The object $I^{\bullet}$ in the extension (3.6) is not quasi-isomorphic to a two-term complex $(\mathcal{E} \rightarrow F)$ for any torsion-free sheaf $\mathcal{E}$ and a one- or zero-dimensional sheaf $F$.

Proof. Suppose to the contrary that $I^{\bullet}$ is quasi-isomorphic to $(\mathcal{E} \stackrel{s}{\rightarrow} F)$. Then $\mathcal{H}^{0}\left(I^{\bullet}\right)=\mathcal{U}=$ $\operatorname{Ker}(s)$, so in particular we have $\mathcal{U} \subset \mathcal{E}$. As $\mathcal{U}$ is reflexive, $\mathcal{E}$ is torsion free, and the inclusion $\mathcal{U} \subset \mathcal{E}$ is isomorphic in codimension one, we have $\mathcal{U}=\mathcal{E}$. Then $F$ and $\mathcal{O}_{x}$ have the same Chern characters, so $F$ is of the form $\mathcal{O}_{y}$ for some point $y \in X$. As $I^{\bullet}$ is not a sheaf, the morphism $s: \mathcal{U} \rightarrow \mathcal{O}_{y}$ must be the zero map. So $I^{\bullet}$ is isomorphic to $\mathcal{U} \oplus \mathcal{O}_{y}[-1]$, and comparing with (3.6), we see that $y=x$ and the sequence (3.6) must split. This gives a contradiction.

Let $M_{\mathrm{PT}}(r, D,-\beta,-n)$ be the moduli space of PT semistable objects in $D^{b} \operatorname{Coh}(X)$ with Chern character (3.1). By the coprime condition (3.2), the space $M_{\mathrm{PT}}(r, D,-\beta,-n)$ consists of only PT stable objects. By [Lo13], it is a proper algebraic space of finite type. Because of the CY3 condition on $X$, it is also equipped with a symmetric perfect obstruction theory [HT10] and the associated zero-dimensional virtual fundamental class. Similarly to (3.3), we can define the invariant

$$
\mathrm{PT}(r, D,-\beta,-n):=\int_{\left[M_{\mathrm{PT}}(r, D,-\beta,-n)\right]^{\mathrm{vir}}} 1 .
$$

Also similarly to (3.4), we consider the generating series

$$
\operatorname{PT}_{r, D}(q, t):=\sum_{\beta, n} \mathrm{PT}(r, D,-\beta,-n) q^{n} t^{\beta}
$$

\subsection{Tilting of $\operatorname{Coh}(X)$}

Recall that a torsion pair on an abelian category $\mathcal{A}$ is a pair of full subcategories $(\mathcal{T}, \mathcal{F})$ on $\mathcal{A}$ such that

(1) we have $\operatorname{Hom}(T, F)=0$ for $T \in \mathcal{T}$ and $F \in \mathcal{F}$;

(2) for any $E \in \mathcal{A}$, there is an exact sequence $0 \rightarrow T \rightarrow E \rightarrow F \rightarrow 0$ with $T \in \mathcal{T}$ and $F \in \mathcal{F}$.

The category $\mathcal{T}$ is called the torsion part of the torsion pair $(\mathcal{T}, \mathcal{F})$. For a torsion pair $(\mathcal{T}, \mathcal{F})$ on $\mathcal{A}$, its tilting is defined by

$$
\mathcal{A}^{\dagger}:=\langle\mathcal{F}, \mathcal{T}[-1]\rangle \subset D^{b}(\mathcal{A}) .
$$

Here $\langle *\rangle$ means the extension closure. The tilting $\mathcal{A}^{\dagger}$ is known to be the heart of a bounded t-structure on $D^{b}(\mathcal{A})$ (cf. [HRS96]). More generally, we introduce the following notion.

Definition 3.6. Let $\mathcal{A}$ be an exact category and $\mathcal{F}_{1}, \mathcal{F}_{2}, \ldots, \mathcal{F}_{n}$ full subcategories of $\mathcal{A}$. Then we write

$$
\mathcal{A}=\left\langle\mathcal{F}_{1}, \mathcal{F}_{2}, \ldots, \mathcal{F}_{n}\right\rangle
$$

if the following conditions are satisfied:

(i) We have $\operatorname{Hom}\left(F_{i}, F_{j}\right)=0$ for $F_{i} \in \mathcal{F}_{i}$ and $F_{j} \in \mathcal{F}_{j}$ with $i<j$.

(ii) For any $E \in \mathcal{A}$, there is a filtration

$$
0=E_{0} \subset E_{1} \subset \cdots \subset E_{n}=E
$$

in $\mathcal{A}$ such that $E_{i} / E_{i-1} \in \mathcal{F}_{i}$. 


\section{Y. TODA}

Remark 3.7. In Definition 3.6, the $n=2$ case corresponds to the torsion pair. As for the torsion pair, the filtration (3.9) is unique if it exists. The filtration (3.9) is an analogue of the HarderNarasimhan filtration in some stability condition. Also, for any $1 \leqslant i \leqslant n$, we have the torsion pair in $\mathcal{A}$

$$
\mathcal{A}=\left\langle\left\langle\mathcal{F}_{1}, \ldots, \mathcal{F}_{i}\right\rangle,\left\langle\mathcal{F}_{i+1}, \ldots, \mathcal{F}_{n}\right\rangle\right\rangle
$$

Let $(X, \omega)$ be as in the previous subsections. For an interval $I \subset \mathbb{R} \cup\{\infty\}$, we set

$$
\operatorname{Coh}_{I}(X):=\left\langle E \in \operatorname{Coh}(X): E \text { is } \mu_{\omega} \text {-semistable with } \mu_{\omega}(E) \in I\right\rangle \cup\{0\} .
$$

Let us take the numerical class as in (3.1). We set

$$
\mu:=\frac{D \cdot \omega^{2}}{r} \in \mathbb{Q} .
$$

By the existence of Harder-Narasimhan filtrations with respect to the $\mu_{\omega}$-stability, we have the following torsion pair in $\operatorname{Coh}(X)$ :

$$
\operatorname{Coh}(X)=\left\langle\operatorname{Coh}_{>\mu}(X), \operatorname{Coh}_{\leqslant \mu}(X)\right\rangle .
$$

We take its tilting $\mathcal{A}_{\mu}:=\left\langle\operatorname{Coh}_{\leqslant \mu}(X), \operatorname{Coh}_{>\mu}(X)[-1]\right\rangle$. By construction, for any $E \in \mathcal{A}_{\mu}$, we have $\operatorname{rank}(E) \cdot D \omega^{2}-c_{1}(E) \omega^{2} \cdot r \geqslant 0$.

Therefore, the category $\mathcal{B}_{\mu}:=\left\{E \in \mathcal{A}_{\mu}: \operatorname{rank}(E) \cdot D \omega^{2}-c_{1}(E) \omega^{2} \cdot r=0\right\}$ is an abelian subcategory of $\mathcal{A}_{\mu}$. From the construction of $\mathcal{A}_{\mu}$, it is easy to see that

$$
\mathcal{B}_{\mu}=\left\langle\operatorname{Coh}_{\mu}(X), \operatorname{Coh}_{\leqslant 1}(X)[-1]\right\rangle .
$$

Here $\operatorname{Coh}_{\leqslant 1}(X)$ is the category of sheaves $F$ with $\operatorname{dim} \operatorname{Supp}(F) \leqslant 1$. Let $\bar{\mu}_{\omega}$ be the slope function on $\mathrm{Coh}_{\leqslant 1}(X)$ defined by

$$
\bar{\mu}_{\omega}(F):=\frac{\operatorname{ch}_{3}(F)}{\operatorname{ch}_{2}(F) \cdot \omega} .
$$

Similarly to the $\mu_{\omega}$-stability, the above slope function on $\operatorname{Coh}_{\leqslant 1}(X)$ defines the $\bar{\mu}_{\omega}$-stability on $\mathrm{Coh}_{\leqslant 1}(X)$. For any interval $I \subset \mathbb{R} \cup\{\infty\}$, we set

$$
\mathcal{C}_{I}:=\left\langle F \in \operatorname{Coh}_{\leqslant 1}(X): F \text { is } \bar{\mu}_{\omega} \text {-semistable with } \bar{\mu}_{\omega}(F) \in I\right\rangle[-1] \cup\{0\} .
$$

Then using the notation in Definition 3.6, we can write $\mathcal{B}_{\mu}$ as

$$
\mathcal{B}_{\mu}=\left\langle\operatorname{Coh}_{\mu}(X), \mathcal{C}_{\infty}, \mathcal{C}_{[0, \infty)}, \mathcal{C}_{<0}\right\rangle
$$

Note that $\mathcal{C}_{\infty}$ consists of $Q[-1]$ for zero-dimensional sheaves $Q$. We will consider its subcategory

$$
\mathcal{D}_{\mu}:=\left\langle\operatorname{Coh}_{\mu}(X), \mathcal{C}_{\infty}, \mathcal{C}_{[0, \infty)}\right\rangle .
$$

Lemma 3.8. The subcategories $\mathcal{D}_{\mu},\left\langle\operatorname{Coh}_{\mu}(X), \mathcal{C}_{\infty}\right\rangle, \mathcal{C}_{\infty}, \mathcal{C}_{[0, \infty]}$ in $\mathcal{B}_{\mu}$ are closed under quotients in the abelian category $\mathcal{B}_{\mu}$.

Proof. The categories $\mathcal{D}_{\mu}$ and $\left\langle\operatorname{Coh}_{\mu}(X), \mathcal{C}_{\infty}\right\rangle$ are closed under quotients since they are torsion parts of some torsion pairs of $\mathcal{B}_{\mu}$. Since $E \in \mathcal{B}_{\mu}$ has $\operatorname{rank}(E)$ at least 0 with $\operatorname{rank}(E)=0$ if and only if $E \in \mathrm{Coh}_{\leqslant 1}(X)[-1]$, we see that $\mathrm{Coh}_{\leqslant 1}(X)[-1]$ is closed under quotients and subobjects in $\mathcal{B}_{\mu}$. Then the categories $\mathcal{C}_{\infty}$ and $\mathcal{C}_{[0, \infty]}$ are also closed under quotients in $\mathcal{B}_{\mu}$ since they are also torsion parts of some torsion pairs of $\mathrm{Coh}_{\leqslant 1}(X)[-1]$.

Remark 3.9. Note that both $\mu_{\omega}$-semistable objects and PT semistable objects with Chern character (3.1) are objects in $\mathcal{D}_{\mu}$. Indeed, they are contained in the smaller subcategories $\operatorname{Coh}_{\mu}(X)$ and $\left\langle\operatorname{Coh}_{\mu}(X), \mathcal{C}_{\infty}\right\rangle$, respectively. 


\section{HALL ALGEBRAS}

\subsection{Completions of Hall algebras}

It is known that the stack of objects in $\mathcal{A}_{\mu}$ forms an open substack of $\mathcal{M}$ (cf. [PT19, Proposition 4.11]). Therefore, by Section 2.6, we can define the Hall algebra $H\left(\mathcal{A}_{\mu}\right)$ of $\mathcal{A}_{\mu}$, the associated Poisson algebra $H^{\mathrm{sc}}\left(\mathcal{A}_{\mu}\right)$, and the Poisson algebra homomorphism

$$
I: H^{\mathrm{sc}}\left(\mathcal{A}_{\mu}\right) \rightarrow C(X) .
$$

We construct certain completions of these Hall algebras using some inequalities of Chern characters. First, for any torsion-free $\mu_{\omega}$-semistable sheaf $E$ on $X$, we have the Bogomolov inequality

$$
\left(\operatorname{ch}_{1}(E) \omega^{2}\right)^{2} \geqslant 2 \operatorname{ch}_{0}(E) \omega^{3} \cdot \operatorname{ch}_{2}(E) \omega .
$$

Second, by Langer [Lan09, Section 3], there is a function

$$
l: H^{0}(X) \oplus H^{2}(X) \oplus H^{4}(X) \rightarrow \mathbb{Q}_{>0}
$$

such that any torsion-free $\mu_{\omega}$-semistable sheaf $E$ satisfies

$$
\operatorname{ch}_{3}(E) \leqslant l\left(\operatorname{ch}_{0}(E), \operatorname{ch}_{1}(E), \operatorname{ch}_{2}(E)\right) .
$$

For a fixed $(r, D) \in H^{0}(X) \oplus H^{2}(X)$ satisfying (3.2), we define

$$
\Gamma_{r, D}:=\left\{(r, D,-\beta,-n) \in \Gamma: \omega \beta \geqslant-\frac{\left(D \omega^{2}\right)^{2}}{2 r \omega^{3}}, n \geqslant-l(r, D,-\beta)\right\} .
$$

We also define

$$
\Gamma_{\sharp}:=\{(0,0,-\beta,-n) \in \Gamma: \beta \geqslant 0, n \geqslant 0\} .
$$

Here, $\beta>0$ means that $\beta$ is the Poincaré dual of an effective algebraic one-cycle on $X$. We have the following obvious lemma.

Lemma 3.10. (i) For any $E \in \mathcal{D}_{\mu}$ with $\left(\operatorname{ch}_{0}(E), \operatorname{ch}_{1}(E)\right)=(r, D)$, we have $\operatorname{ch}(E) \in \Gamma_{r, D}$.

(ii) For any $F \in \mathcal{D}_{\mu}$ with $\operatorname{ch}_{0}(F)=0$, we have $\operatorname{ch}(F) \in \Gamma_{\sharp}$.

(iii) For $v \in \Gamma_{r, D}$ and $v^{\prime} \in \Gamma_{\sharp}$, we have $v+v^{\prime} \in \Gamma_{r, D}$.

(iv) For $v \in \Gamma_{\sharp}$, there are only a finite number of ways to write $v$ as $v_{1}+v_{2}+\cdots+v_{l}$ for $v_{1}, \ldots, v_{l} \in \Gamma_{\sharp} \backslash\{0\}$.

(v) For $v \in \Gamma_{r, D}$, there are only a finite number of ways to write $v$ as $v_{1}+v_{2}+\cdots+v_{l}+v_{l+1}$ for $v_{1}, \ldots, v_{l} \in \Gamma_{\sharp} \backslash\{0\}$ and $v_{l+1} \in \Gamma_{r, D}$.

Lemma 3.10 also implies the following lemma, which will be used in Section 4.2.

Lemma 3.11. The set of objects in $\mathcal{D}_{\mu}$ with a fixed Chern character is bounded.

Proof. The result follows from Lemma 3.10 together with the fact that the set of semistable sheaves with a fixed Chern character is bounded.

We set

$$
\widehat{H}_{r, D}\left(\mathcal{A}_{\mu}\right):=\prod_{v \in \Gamma_{r, D}} H_{v}\left(\mathcal{A}_{\mu}\right), \quad \widehat{H}_{\sharp}\left(\mathcal{A}_{\mu}\right):=\prod_{v \in \Gamma_{\sharp}} H_{v}\left(\mathcal{A}_{\mu}\right) .
$$

By Lemma 3.10, the Hall product on $H\left(\mathcal{A}_{\mu}\right)$ induces one on $\widehat{H}_{\sharp}\left(\mathcal{A}_{\mu}\right)$, and $\widehat{H}_{r, D}\left(\mathcal{A}_{\mu}\right)$ is a bi-module over $\widehat{H}_{\sharp}\left(\mathcal{A}_{\mu}\right)$. As in Section 2.6, we can define the subspaces of regular elements

$$
\widehat{H}_{r, D}^{\mathrm{reg}}\left(\mathcal{A}_{\mu}\right) \subset \widehat{H}_{r, D}\left(\mathcal{A}_{\mu}\right) \quad \text { and } \quad \widehat{H}_{\sharp}^{\mathrm{reg}}\left(\mathcal{A}_{\mu}\right) \subset \widehat{H}_{\sharp}\left(\mathcal{A}_{\mu}\right)
$$




\section{Y. TODA}

such that $\widehat{H}_{\sharp}^{\mathrm{reg}}\left(\mathcal{A}_{\mu}\right)$ is a subalgebra of $\widehat{H}_{\sharp}\left(\mathcal{A}_{\mu}\right)$ and $\widehat{H}_{r, D}^{\mathrm{reg}}\left(\mathcal{A}_{\mu}\right)$ is a bi-module over $\widehat{H}_{\sharp}^{\mathrm{reg}}\left(\mathcal{A}_{\mu}\right)$. We can also define the quotient spaces

$$
\begin{aligned}
\widehat{H}_{r, D}^{\mathrm{sc}}\left(\mathcal{A}_{\mu}\right) & =\widehat{H}_{r, D}^{\mathrm{reg}}\left(\mathcal{A}_{\mu}\right) /(\mathbb{L}-1) \cdot \widehat{H}_{r, D}^{\mathrm{reg}}\left(\mathcal{A}_{\mu}\right), \\
\widehat{H}_{\sharp}^{\mathrm{sc}}\left(\mathcal{A}_{\mu}\right) & =\widehat{H}_{\sharp}^{\mathrm{reg}}\left(\mathcal{A}_{\mu}\right) /(\mathbb{L}-1) \cdot \widehat{H}_{\sharp}^{\mathrm{reg}}\left(\mathcal{A}_{\mu}\right)
\end{aligned}
$$

such that we have the induced Poisson algebra structure on $\widehat{H}_{\sharp}^{\mathrm{sc}}\left(\mathcal{A}_{\mu}\right)$ and $\widehat{H}_{r, D}^{\mathrm{sc}}\left(\mathcal{A}_{\mu}\right)$ is a Poisson bi-module over $\widehat{H}_{\sharp}^{\mathrm{sc}}\left(\mathcal{A}_{\mu}\right)$.

Remark 3.12. For $F \in \mathcal{A}_{\mu}$, it is easy to see that $\operatorname{ch}(F) \in \Gamma_{\sharp}$ if and only if $F \in \operatorname{Coh}_{\leqslant 1}(X)[-1]$. Hence, we can also write $\widehat{H}_{\sharp}\left(\mathcal{A}_{\mu}\right)$ as

$$
\widehat{H}_{\sharp}\left(\mathcal{A}_{\mu}\right)=\prod_{v \in \Gamma_{\sharp}} H_{v}\left(\operatorname{Coh}_{\leqslant 1}(X)[-1]\right) .
$$

By Lemma 3.10 again, for any $\gamma \in \widehat{H}_{\sharp}\left(\mathcal{A}_{\mu}\right)$ with zero $H_{0}\left(\mathcal{A}_{\mu}\right)$-component, we have the welldefined elements

$$
\exp (\gamma), \log (1+\gamma),(1+\gamma)^{-1} \in \widehat{H}_{\sharp}\left(\mathcal{A}_{\mu}\right)
$$

We also set

$$
\widehat{C}_{r, D}(X):=\prod_{v \in \Gamma_{r, D}} \mathbb{Q} \cdot c_{v} \quad \text { and } \quad \widehat{C}_{\sharp}(X):=\prod_{v \in \Gamma_{\sharp}} \mathbb{Q} \cdot c_{v} .
$$

As above, $\widehat{C}_{\sharp}(X)$ is a Poisson algebra and $\widehat{C}_{r, D}(X)$ is a Poisson bi-module over $\widehat{C}_{\sharp}(X)$. The integration map (3.14) induces the maps

$$
I_{r, D}: \widehat{H}_{r, D}\left(\mathcal{A}_{\mu}\right) \rightarrow \widehat{C}_{r, D}(X) \quad \text { and } \quad I_{\sharp}: \widehat{H}_{\sharp}\left(\mathcal{A}_{\mu}\right) \rightarrow \widehat{C}_{\sharp}(X)
$$

such that $I_{\sharp}$ is a Poisson algebra homomorphism and $I_{r, D}$ is a Poisson bi-module homomorphism over $\widehat{H}_{\sharp}\left(\mathcal{A}_{\mu}\right)$.

\subsection{Elements of the Hall algebra}

Let $\mathcal{M}_{\mathrm{DT}}(r, D)$ and $\mathcal{M}_{\mathrm{PT}}(r, D)$ be the stacks of $\mu_{\omega}$-stable sheaves, PT stable objects, respectively.

Lemma 3.13. The stacks $\mathcal{M}_{\mathrm{DT}}(r, D)$ and $\mathcal{M}_{\mathrm{PT}}(r, D)$ are $\mathbb{C}^{*}$-gerbes over the unions of the moduli spaces $M_{\mathrm{DT}}(r, D,-\beta,-n)$ and $M_{\mathrm{PT}}(r, D,-\beta,-n)$, respectively, for all possible $(\beta, n)$.

Proof. Since any $[E] \in \mathcal{M}_{\mathrm{DT}}(r, D)$ is a stable sheaf by the coprime condition (3.2), we have $\operatorname{Aut}(E)=\mathbb{C}^{*}$. Similarly, any $I^{\bullet} \in \mathcal{M}_{\mathrm{PT}}(r, D)$ is a stable object with respect to a certain polynomial stability condition [Lo12]; we also have $\operatorname{Aut}\left(I^{\bullet}\right)=\mathbb{C}^{*}$. Hence, the lemma follows.

Lemma 3.13 in particular implies that all connected components of $\mathcal{M}_{\mathrm{DT}}(r, D)$ and $\mathcal{M}_{\mathrm{PT}}(r, D)$ are of finite type. Also, by Remark 3.9 and Lemma 3.10(i), the Chern characters of objects in $\mathcal{M}_{\mathrm{DT}}(r, D)$ and $\mathcal{M}_{\mathrm{PT}}(r, D)$ are contained in $\Gamma_{r, D}$. Therefore, for $\star \in\{\mathrm{DT}, \mathrm{PT}\}$, we obtain the elements

$$
\delta_{\star}(r, D):=\left[\mathcal{M}_{\star}(r, D) \rightarrow \mathcal{O} b j\left(\mathcal{A}_{\mu}\right)\right] \in \widehat{H}_{r, D}\left(\mathcal{A}_{\mu}\right)
$$

We also set

$$
\bar{\delta}_{\star}(r, D):=(\mathbb{L}-1) \cdot \delta_{\star}(r, D) .
$$




\section{HALL ALGEBRAS}

Then Lemma 3.13 implies that

$$
\bar{\delta}_{\star}(r, D)=\sum_{v \in \Gamma_{r, D}}\left[M_{\star}(v) \rightarrow \mathcal{O} b j\left(\mathcal{A}_{\mu}\right)\right] \in \widehat{H}_{r, D}^{\mathrm{reg}}\left(\mathcal{A}_{\mu}\right) .
$$

Here, $M_{\star}(v)$ denotes the moduli space $M_{\mathrm{DT}}(r, D,-\beta,-n)$ for $\star=\mathrm{DT}$ and $M_{\mathrm{PT}}(r, D,-\beta,-n)$ for $\star=\mathrm{PT}$.

In a similar way, for any interval $I \subset \mathbb{R}_{\geqslant 0} \cup\{\infty\}$, the stack of objects $\mathcal{O b j}\left(\mathcal{C}_{I}\right)$ in $\mathcal{C}_{I}$ decomposes into

$$
\mathcal{O b j}\left(\mathcal{C}_{I}\right)=\coprod_{v \in \Gamma_{\sharp}, \bar{\mu}_{\omega}(v) \in I, v \neq 0} \mathcal{O} b j_{v}\left(\mathcal{C}_{I}\right)
$$

such that each component $\mathcal{O} b j_{v}\left(\mathcal{C}_{I}\right)$ is a finite-type stack. Here, we have used the map

$$
\bar{\mu}_{\omega}: \Gamma_{\sharp} \backslash\{0\} \rightarrow \mathbb{Q} \geqslant 0 \cup\{\infty\}, \quad(\beta, n) \mapsto \frac{n}{\omega \cdot \beta} .
$$

Hence, we have the element

$$
\delta\left(\mathcal{C}_{I}\right):=\left[\mathcal{O b j}\left(\mathcal{C}_{I}\right) \rightarrow \mathcal{O} b j\left(\mathcal{A}_{\mu}\right)\right] \in \widehat{H}_{\sharp}\left(\mathcal{A}_{\mu}\right) .
$$

Substituting (3.15), we obtain the element

$$
\epsilon\left(\mathcal{C}_{I}\right):=\log \left(\delta\left(\mathcal{C}_{I}\right)\right) \in \widehat{H}_{\sharp}\left(\mathcal{A}_{\mu}\right) .
$$

The following is an important consequence of Joyce's absence of poles result.

Theorem 3.14 ([Joy07, Theorem 8.7], [Bri11, Theorem 6.3]). We have

$$
\bar{\epsilon}\left(\mathcal{C}_{I}\right):=(\mathbb{L}-1) \cdot \epsilon\left(\mathcal{C}_{I}\right) \in \widehat{H}_{\sharp}^{\mathrm{reg}}\left(\mathcal{A}_{\mu}\right) .
$$

\subsection{Applications of the integration map}

Let us project elements $\bar{\delta}_{\star}(r, D)$ to $\widehat{H}_{r, D}^{\text {sc }}\left(\mathcal{A}_{\mu}\right)$ and apply the integration map (3.16). Then we have the following relations:

$$
\begin{aligned}
& I_{r, D}\left(\bar{\delta}_{\mathrm{DT}}(r, D)\right)=-\sum_{v \in \Gamma_{r, D}} \mathrm{DT}(v) \cdot c_{v}, \\
& I_{r, D}\left(\bar{\delta}_{\mathrm{PT}}(r, D)\right)=-\sum_{v \in \Gamma_{r, D}} \mathrm{PT}(v) \cdot c_{v} .
\end{aligned}
$$

Here, we have used Behrend's result [Beh09] describing virtual classes associated with symmetric obstruction theories by the integrations of his constructible functions.

Next, we project elements $\bar{\epsilon}\left(\mathcal{C}_{I}\right)$ to $\widehat{H}_{\sharp}^{\mathrm{sc}}\left(\mathcal{A}_{\mu}\right)$ and apply the integration map (3.16). For $\bar{\mu} \in$ $\mathbb{Q} \geqslant 0 \cup\{\infty\}$ and a non-zero $v \in \Gamma_{\sharp}$ with $\bar{\mu}_{\omega}(v)=\bar{\mu}$, we obtain the invariant $N_{v} \in \mathbb{Q}$ given by the following formula:

$$
I_{\sharp}\left(\bar{\epsilon}\left(\mathcal{C}_{\bar{\mu}}\right)\right)=-\sum_{0 \neq v \in \Gamma_{\sharp}, \bar{\mu}_{\omega}(v)=\bar{\mu}} N_{v} \cdot c_{v} .
$$

Below, for $(0,0,-\beta,-n) \in \Gamma_{\sharp}$, we write

$$
N_{n, \beta}:=N_{(0,0,-\beta,-n)} \in \mathbb{Q} .
$$

Remark 3.15. The invariant $N_{n, \beta}$ is a virtual count of $\bar{\mu}_{\omega}$-semistable sheaves $F \in \operatorname{Coh}_{\leqslant 1}(X)$ with $[F]=\beta$ and $\chi(F)=n$, which plays an important role in the wall-crossing of curve-counting 


\section{Y. TODA}

theory; see, for example, [Tod12, Definition 4.7]. In particular, if $N_{n, \beta} \neq 0$, then $\beta$ is either zero or a Poincaré dual of an effective one-cycle on $X$, and $n \in \mathbb{Z}_{\geqslant 0}$.

The following fact is also standard, but we include the proof for completeness.

Lemma 3.16. For any interval $I \subset \mathbb{R}_{\geqslant 0} \cup\{\infty\}$, we have

$$
I_{\sharp}\left(\bar{\epsilon}\left(\mathcal{C}_{I}\right)\right)=-\sum_{0 \neq v \in \Gamma_{\sharp}, \bar{\mu}_{\omega}(v) \in I} N_{v} \cdot c_{v} .
$$

Proof. By the existence of Harder-Narasimhan filtrations with respect to the $\bar{\mu}_{\omega}$-stability, we have the identity in $\widehat{H}_{\sharp}\left(\mathcal{A}_{\mu}\right)$ (cf. [Joy08, Theorem 5.11])

$$
\delta\left(\mathcal{C}_{I}\right)=\prod_{\bar{\mu} \in I} \delta\left(\mathcal{C}_{\bar{\mu}}\right) .
$$

On the right-hand side, we take the product with the decreasing order of $\bar{\mu}$. By taking the logarithm of both sides and multiplying by $(\mathbb{L}-1)$, we obtain the identity in $\widehat{H}_{\sharp}^{\text {sc }}\left(\mathcal{A}_{\mu}\right)$

$$
\bar{\epsilon}\left(\mathcal{C}_{I}\right)=\sum_{\bar{\mu} \in I} \bar{\epsilon}\left(\mathcal{C}_{\bar{\mu}}\right)+\left\{\text { nested Poisson brackets in } \bar{\epsilon}\left(\mathcal{C}_{\bar{\mu}}\right)\right\} .
$$

Since $\chi\left(v_{1}, v_{2}\right)=0$ for $v_{i} \in \Gamma_{\sharp}$, the property of the integration map $I_{\sharp}$ shows that

$$
I_{\sharp}\left(\bar{\epsilon}\left(\mathcal{C}_{I}\right)\right)=\sum_{\bar{\mu} \in I} I_{\sharp}\left(\bar{\epsilon}\left(\mathcal{C}_{\bar{\mu}}\right)\right) .
$$

By (3.22), we obtain the desired identity.

\subsection{Proof of Theorem $\mathbf{1 . 2}$}

We denote by $\operatorname{Coh}_{\mu}^{P}(X) \subset D^{b} \operatorname{Coh}(X)$ the category of PT semistable objects $I^{\bullet}$ with $\mu_{\omega}\left(I^{\bullet}\right)=\mu$. Let $\mathcal{B}_{\mu}$ be the abelian category introduced in (3.12).

Lemma 3.17. We have the following identity in $\mathcal{B}_{\mu}$ :

$$
\left\langle\operatorname{Coh}_{\mu}(X), \mathcal{C}_{\infty}\right\rangle=\left\langle\mathcal{C}_{\infty}, \operatorname{Coh}_{\mu}^{P}(X)\right\rangle .
$$

Proof. By the definition of PT semistable objects, we have

$$
\operatorname{Coh}_{\mu}^{P}(X)=\left\{E \in\left\langle\operatorname{Coh}_{\mu}(X), \mathcal{C}_{\infty}\right\rangle: \operatorname{Hom}\left(\mathcal{C}_{\infty}, E\right)=0\right\}
$$

It is enough to show that any object $E$ on the left-hand side of (3.24) fits into an exact sequence

$$
0 \rightarrow T \rightarrow E \rightarrow F \rightarrow 0
$$

in $\mathcal{B}_{\mu}$ with $T \in \mathcal{C}_{\infty}$ and $F \in \operatorname{Coh}_{\mu}^{P}(X)$. Suppose $E \notin \operatorname{Coh}_{\mu}^{P}(X)$. Then there is a non-zero morphism $T \rightarrow E$ for some $T \in \mathcal{C}_{\infty}$. By Lemma 3.8, the object $\mathcal{C}_{\infty}$ is closed under quotients in $\mathcal{B}_{\mu}$, so we may assume that $T \rightarrow E$ is injective in $\mathcal{B}_{\mu}$. Then the quotient $F=E / T$ in $\mathcal{B}_{\mu}$ is an object on the left-hand side of (3.24) by Lemma 3.8 again. Because $\mathcal{A}_{\mu}$ is noetherian (cf. the proof of [BMT14, Lemma 3.2.4]), the abelian category $\mathcal{B}_{\mu}$ is also noetherian, so this process must terminate. Hence we obtain the exact sequence (3.25).

Let us consider the series $\mathrm{DT}_{r, D}(q, t)$ and $\mathrm{PT}_{r, D}(q, t)$ introduced in (3.4) and (3.8). We are now ready to prove Theorem 1.2. 
Theorem 3.18. We have the following identity:

$$
\mathrm{DT}_{r, D}(q, t)=M\left((-1)^{r} q\right)^{r \cdot e(X)} \cdot \mathrm{PT}_{r, D}(q, t) .
$$

Proof. By Lemma 3.17, we have the following identity in $\widehat{H}_{r, D}\left(\mathcal{A}_{\mu}\right)$ :

$$
\delta_{\mathrm{DT}}(r, D) * \delta\left(\mathcal{C}_{\infty}\right)=\delta\left(\mathcal{C}_{\infty}\right) * \delta_{\mathrm{PT}}(r, D) .
$$

Using (3.19), we obtain the identity

$$
\delta_{\mathrm{DT}}(r, D)=\exp \left(\epsilon\left(\mathcal{C}_{\infty}\right)\right) * \delta_{\mathrm{PT}}(r, D) * \exp \left(-\epsilon\left(\mathcal{C}_{\infty}\right)\right) .
$$

For $a \in \widehat{H}_{\sharp}\left(\mathcal{A}_{\mu}\right)$, its adjoint action on $\widehat{H}_{r, D}\left(\mathcal{A}_{\mu}\right)$ is denoted by

$$
\operatorname{Ad}(a) \circ x:=a * x-x * a: \widehat{H}_{r, D}\left(\mathcal{A}_{\mu}\right) \rightarrow \widehat{H}_{r, D}\left(\mathcal{A}_{\mu}\right) .
$$

Applying the Baker-Campbell-Hausdorff formula to (3.26), we obtain

$$
\delta_{\mathrm{DT}}(r, D)=\exp \left(\operatorname{Ad}\left(\epsilon\left(\mathcal{C}_{\infty}\right)\right)\right) \circ \delta_{\mathrm{PT}}(r, D) .
$$

We multiply both sides by $(\mathbb{L}-1)$ and project them to $\widehat{H}_{r, D}^{\mathrm{sc}}\left(\mathcal{A}_{\mu}\right)$. By writing the adjoint action of $a \in \widehat{H}_{\sharp}^{\mathrm{sc}}\left(\mathcal{A}_{\mu}\right)$ as

$$
\operatorname{Ad}^{\mathrm{sc}}(a) \circ x:=\{a, x\}: \widehat{H}_{r, D}^{\mathrm{sc}}\left(\mathcal{A}_{\mu}\right) \rightarrow \widehat{H}_{r, D}^{\mathrm{sc}}\left(\mathcal{A}_{\mu}\right),
$$

we obtain the following identity in $\widehat{H}_{r, D}^{\mathrm{sc}}\left(\mathcal{A}_{\mu}\right)$ :

$$
\bar{\delta}_{\mathrm{DT}}(r, D)=\exp \left(\operatorname{Ad}^{\mathrm{sc}}\left(\bar{\epsilon}\left(\mathcal{C}_{\infty}\right)\right)\right) \circ \bar{\delta}_{\mathrm{PT}}(r, D) .
$$

By applying the integration map $I_{r, D}$ in (3.16) and using (3.22) and the computation (2.9), we obtain the formula

$$
\mathrm{DT}_{r, D}(q, t)=\exp \left(\sum_{n>0}(-1)^{r n-1} r n N_{n, 0} q^{n}\right) \cdot \mathrm{PT}_{r, D}(q, t) .
$$

By substituting $(r, D)=(1,0)$ and $t=0$ and comparing with the computation of the series $\mathrm{DT}_{(1,0)}(q, 0)$ in $[\mathrm{BF} 08, \mathrm{LP} 09, \mathrm{Li} 06]$, we obtain

$$
\exp \left(\sum_{n>0}(-1)^{n-1} n N_{n, 0} q^{n}\right)=M(-q)^{e(X)} .
$$

Therefore, we obtain the desired identity.

\section{Rationality of higher-rank PT invariants}

In this section, we prove Theorem 1.3.

\subsection{The category $\operatorname{Coh}_{\mu}^{L}(X)$}

As before, let $\mu$ be the rational number $D \cdot \omega^{2} / r$ introduced in (3.10) and $\mathcal{D}_{\mu}$ be the category (3.13). We define the category

$$
\operatorname{Coh}_{\mu}^{L}(X):=\left\{E \in \mathcal{D}_{\mu}: \operatorname{Hom}(F, E)=0 \text { for any } F \in \mathcal{C}_{[0, \infty]}\right\}
$$

We prepare some lemmas on this category.

Lemma 4.1. We have the identity $\mathcal{D}_{\mu}=\left\langle\mathcal{C}_{[0, \infty]}, \operatorname{Coh}_{\mu}^{L}(X)\right\rangle$. 


\section{Y. TODA}

Proof. Using Lemma 3.8 and replacing $\left\langle\operatorname{Coh}_{\mu}(X), \mathcal{C}_{\infty}\right\rangle, \mathcal{C}_{\infty}, \operatorname{Coh}_{\mu}^{P}(X)$ with $\mathcal{D}_{\mu}, \mathcal{C}_{[0, \infty]}, \operatorname{Coh}_{\mu}^{L}(X)$, respectively, in the proof of Lemma 3.17, we obtain the desired identity.

Lemma 4.2. For any $E \in \operatorname{Coh}_{\mu}^{L}(X)$ with $\left(\operatorname{ch}_{0}(E), \operatorname{ch}_{1}(E)\right)=(r, D)$, we have $\operatorname{Aut}(E)=\mathbb{C}^{*}$.

Proof. It is enough to show that any non-zero morphism $\phi: E \rightarrow E$ is an isomorphism. Let $0 \neq F \subset E$ be the image of $\phi$ in $\mathcal{B}_{\mu}$ and $G \subset F$ the kernel of $\phi$ in $\mathcal{B}_{\mu}$. Suppose $F \subsetneq E$. Because of the coprime condition (3.2), we have either $\operatorname{rank}(F)=0$ or $\operatorname{rank}(G)=0$. In the $\operatorname{rank}(F)=0$ case, we have $F \in \operatorname{Coh}_{\leqslant 1}(X)[-1]$. On the other hand, we have $F \in \mathcal{D}_{\mu}$ by Lemma 3.8, hence $F \in \mathcal{C}_{[0, \infty]}$. But this contradicts $E \in \operatorname{Coh}_{\mu}^{L}(X)$. In the $\operatorname{rank}(G)=0$ case, the condition $E \in \operatorname{Coh}_{\mu}^{L}(X) \operatorname{implies}$ $G \in \mathcal{C}_{<0}$. Also by Lemma 3.8 , we have $E / F \in \mathcal{C}_{[0, \infty]}$. Since $\operatorname{ch}(G)=\operatorname{ch}(E / F)$, we obtain a contradiction. Therefore, we must have $F=E$. But then $G \in \mathcal{B}_{\mu}$ satisfies $\operatorname{ch}(G)=0$, hence $G=0$. Therefore, $\phi$ is an isomorphism.

By Lemma 4.1, the abelian category $\mathcal{B}_{\mu}$ can be written as

$$
\mathcal{B}_{\mu}=\left\langle\mathcal{C}_{[0, \infty]}, \operatorname{Coh}_{\mu}^{L}(X), \mathcal{C}_{<0}\right\rangle .
$$

Let $\mathbb{D}$ be the dualizing functor on $D^{b} \operatorname{Coh}(X)$ defined by $\mathbb{D}(E):=\mathbf{R} \mathcal{H o m}\left(E, \mathcal{O}_{X}\right)$. The next proposition shows that the category $\operatorname{Coh}_{\mu}^{L}(X)$ behaves well under the duality.

Proposition 4.3. The abelian category $\mathcal{B}_{-\mu}$ can be written as

$$
\mathcal{B}_{-\mu}=\left\langle\mathcal{C}_{(0, \infty]}, \mathbb{D}\left(\operatorname{Coh}_{\mu}^{L}(X)\right), \mathcal{C}_{\leqslant 0}\right\rangle
$$

Proof. Note that $\mathcal{B}_{\mu}$ can also written as $\mathcal{B}_{\mu}=\left\langle\mathcal{C}_{\infty}, \operatorname{Coh}_{\mu}^{P}(X), \mathcal{C}_{<\infty}\right\rangle$ by Lemma 3.17. Let $\mathcal{B}_{\mu}^{\dagger}$ be the tilting at $\mathcal{C}_{\infty}$, that is, $\mathcal{B}_{\mu}^{\dagger}:=\left\langle\operatorname{Coh}_{\mu}^{P}(X), \mathcal{C}_{<\infty}, \mathcal{C}_{\infty}[-1]\right\rangle$. We first claim that $\mathbb{D}$ induces the equivalence

$$
\mathbb{D}: \mathcal{B}_{\mu} \stackrel{\sim}{\rightarrow} \mathcal{B}_{-\mu}^{\dagger}
$$

Indeed, we have $\mathbb{D}\left(\mathcal{C}_{<\infty}\right)=\mathcal{C}_{<\infty}$ and $\mathbb{D}\left(\mathcal{C}_{\infty}\right)=\mathcal{C}_{\infty}[-1]$. Also by [PT19, Lemma 4.16], the following holds:

$$
\mathbb{D}\left(\operatorname{Coh}_{\mu}^{P}(X)\right)=\left\{E \in \mathcal{B}_{-\mu}: \operatorname{Hom}\left(\operatorname{Coh}_{\leqslant 1}(X)[-1], E\right)=0\right\} .
$$

In particular, we have $\mathbb{D}\left(\operatorname{Coh}_{\mu}^{P}(X)\right) \subset \mathcal{B}_{-\mu}^{\dagger}$; therefore, $\mathbb{D}\left(\mathcal{B}_{\mu}\right) \subset \mathcal{B}_{-\mu}^{\dagger}$ holds. A similar argument shows that $\mathbb{D}\left(\mathcal{B}_{-\mu}^{\dagger}\right) \subset \mathcal{B}_{\mu}$; hence, the equivalence (4.3) holds. Applying (4.3) to the description (4.1) and noting that $\mathbb{D}\left(\mathcal{C}_{[0, \infty)}\right)=\mathcal{C}_{\leqslant 0}$ and $\mathbb{D}\left(\mathcal{C}_{<0}\right)=\mathcal{C}_{(0, \infty)}$, we obtain the identity

$$
\mathcal{B}_{-\mu}^{\dagger}=\left\langle\mathcal{C}_{(0, \infty)}, \mathbb{D}\left(\operatorname{Coh}_{\mu}^{L}(X)\right), \mathcal{C}_{\leqslant 0}, \mathcal{C}_{\infty}[-1]\right\rangle .
$$

By tilting $\mathcal{C}_{\infty}[-1]$ back, we obtain the desired identity (4.2).

The following corollary obviously follows from Proposition 4.3:

Corollary 4.4. We have $\mathbb{D}\left(\operatorname{Coh}_{\mu}^{L}(X)\right) \subset \mathcal{D}_{-\mu}$.

\section{$4.2 L$-invariants}

Let $\mathcal{M}_{\mathrm{L}}(r, D) \subset \mathcal{O} b j\left(\mathcal{A}_{\mu}\right)$ be the substack of objects $E \in \operatorname{Coh}_{\mu}^{L}(X)$ with $\left(\operatorname{ch}_{0}(E), \operatorname{ch}_{1}(E)\right)=$ $(r, d)$. We will not seek to prove that $\mathcal{M}_{\mathrm{L}}(r, D)$ is an open substack of finite type. Instead, the definition of $\operatorname{Coh}_{\mu}^{L}(X)$ and Lemma 3.11 easily imply that the $\mathbb{C}$-valued points of $\mathcal{M}_{\mathrm{L}}(r, D)$ on 


\section{HALL ALGEBRAS}

each component $\mathcal{O} b j_{v}\left(\mathcal{A}_{\mu}\right)$ form a constructible subset. Therefore, by using the motivic relation, we obtain the element

$$
\delta_{\mathrm{L}}(r, D):=\left[\mathcal{M}_{\mathrm{L}}(r, D) \subset \mathcal{O} b j\left(\mathcal{A}_{\mu}\right)\right] \in \widehat{H}_{r, D}\left(\mathcal{A}_{\mu}\right) .
$$

Also, Lemma 4.2 implies $\bar{\delta}_{\mathrm{L}}(r, D):=(\mathbb{L}-1) \cdot \delta_{\mathrm{L}}(r, D) \in \widehat{H}_{r, D}^{\text {reg }}\left(\mathcal{A}_{\mu}\right)$. By projecting $\bar{\delta}_{\mathrm{L}}(r, D)$ to $\widehat{H}_{r, D}^{\text {sc }}\left(\mathcal{A}_{\mu}\right)$ and applying $(3.16)$, we obtain the integer-valued invariants $\mathrm{L}(r, D,-\beta,-n) \in \mathbb{Z}$ by the formula

$$
I_{r, D}\left(\bar{\delta}_{\mathrm{L}}(r, D)\right)=-\sum_{v \in \Gamma_{r, D}} \mathrm{~L}(v) \cdot c_{v}
$$

\subsection{Properties of the generating series}

We discuss some properties of the generating series of $N_{n, \beta}$ and $\mathrm{L}(r, D,-\beta,-n)$. For the former invariants, we have the following.

Lemma 4.5. For a fixed $\beta>0$, the generating series

$$
\sum_{n \geqslant 0} N_{n, \beta} q^{n} \text { and } \sum_{n \geqslant 0} n N_{n, \beta} q^{n}
$$

are rational functions in $q$.

Proof. Since the $\bar{\mu}_{\omega}$-stability is preserved under taking the tensor product with a line bundle $\mathcal{L}$ satisfying $c_{1}(\mathcal{L})=\omega$, we have $N_{n, \beta}=N_{n+\beta \cdot \omega, \beta}$. Keeping in mind Remark 3.15 and the inequality $\beta \cdot \omega>0$, we see that the desired property of $\sum_{n \geqslant 0} N_{n, \beta} q^{n}$ and $\sum_{n \geqslant 0} n N_{n, \beta} q^{n}$ is an easy consequence of this periodicity.

Remark 4.6. In fact, $N_{n, \beta}$ further satisfies $N_{n, \beta}=N_{-n, \beta}$, and this fact also shows that the second series in Lemma 4.5 is a rational function that is invariant under $q \leftrightarrow 1 / q$, as proved in [Tod10b, Lemma 4.6]. However, the first series in Lemma 4.5 is not invariant under $q \leftrightarrow 1 / q$.

As for the L-invariants, we have the following.

Lemma 4.7. For a fixed $(r, D, \beta)$, the generating series

$$
\sum_{6 n \in \mathbb{Z}} \mathrm{L}(r, D,-\beta,-n) q^{n}
$$

is a Laurent polynomial in $q^{1 / 6}$.

Proof. Suppose $\mathrm{L}(r, D,-\beta,-n) \neq 0$. Then we have $(r, \pm D,-\beta, \mp n) \in \Gamma_{r, \pm D}$ by Lemma 3.10(i) and Corollary 4.4. Hence the definition of $\Gamma_{r, D}$ implies that for a fixed $(r, D, \beta)$, we have $\mathrm{L}(r, D,-\beta,-n)=0$ for $|n| \gg 0$. This implies that the series in Lemma 4.7 is a Laurent polynomial in $q^{1 / 6}$.

\subsection{Proof of rationality}

We define the generating series

$$
\mathrm{L}_{r, D}(q, t):=\sum_{\beta, n} \mathrm{~L}(r, D,-\beta,-n) q^{n} t^{\beta} .
$$

The following result is a higher-rank version of the product expansion formula given in [Tod10b, Bri11]. 


\section{Y. TODA}

THEOREM 4.8. We have the following product expansion formula:

$$
\mathrm{PT}_{r, D}(q, t)=\mathrm{L}_{r, D}(q, t) \prod_{n \geqslant 0, \beta>0} \exp \left((-1)^{r n+D \beta} N_{n, \beta} q^{n} t^{\beta}\right)^{D \beta-r n}
$$

Proof. The results of Lemmas 3.17 and 4.1 imply that $\left\langle\operatorname{Coh}_{\mu}^{P}(X), \mathcal{C}_{[0, \infty)}\right\rangle=\left\langle\mathcal{C}_{[0, \infty)}, \operatorname{Coh}_{\mu}^{\mathrm{L}}(X)\right\rangle$. We obtain the following identity in $\widehat{H}_{r, D}\left(\mathcal{A}_{\mu}\right)$ :

$$
\delta_{\mathrm{PT}}(r, D) * \delta\left(\mathcal{C}_{[0, \infty)}\right)=\delta\left(\mathcal{C}_{[0, \infty)}\right) * \delta_{\mathrm{L}}(r, D)
$$

Therefore, by the same argument used to deduce (3.27) in the proof of Theorem 3.18, we obtain the following identity in $\widehat{H}_{r, D}^{\mathrm{sc}}\left(\mathcal{A}_{\mu}\right)$ :

$$
\bar{\delta}_{\mathrm{PT}}(r, D)=\exp \left(\operatorname{Ad}^{\mathrm{sc}}\left(\bar{\epsilon}\left(\mathcal{C}_{[0, \infty)}\right)\right)\right) \circ \bar{\delta}_{\mathrm{L}}(r, D) .
$$

By applying the integration map $I_{r, D}$ in (3.16) and using Lemma 3.16 and the computation (2.9), we obtain the formula

$$
\operatorname{PT}_{r, D}(q, t)=\mathrm{L}_{r, D}(q, t) \exp \left(\sum_{n \geqslant 0, \beta>0}(-1)^{r n+D \beta-1}(r n-D \beta) N_{n, \beta} q^{n} t^{\beta}\right) .
$$

Therefore, we obtain the desired result.

The result of Theorem 1.3 is a corollary of the above result.

Corollary 4.9. Theorem 1.3 holds.

Proof. This is a consequence of the formula (4.4) together with Lemmas 4.5 and 4.7.

\section{ACKNOWLEDGEMENTS}

This article was written while the author was visiting to Massachusetts Institute of Technology in 2016. I am grateful to Davesh Maulik for valuable discussions and to the referee for carefully reading the paper and for making several comments.

\section{REFERENCES}

AHR15 J. Alper, J. Hall, and D. Rydh, A Luna étale slice theorem for algeraic stacks, 2015, arXiv: 1504.06467.

Bay09 A. Bayer, Polynomial Bridgeland stability conditions and the large volume limit, Geom. Topol. 13 (2009), no. 4, 2389-2425; doi:10.2140/gt.2009.13.2389.

BBBJ15 O. Ben-Bassat, C. Brav, V. Bussi, and D. Joyce, A 'Darboux theorem' for shifted symplectic structures on derived Artin stacks, with applications, Geom. Topol. 19 (2015), no. 3, 1287-1359; doi: 10.2140/gt.2015.19.1287.

BCR18 S. V. Beentjes, J. Calabrese, and J.V. Rennemo, A proof of the Donaldson-Thomas crepant resolution conjecture, 2018, arXiv:1810.06581.

Beh09 K. Behrend, Donaldson-Thomas type invariants via microlocal geometry, Ann. of Math. 170 (2009), no. 3, 1307-1338; doi:10.4007/annals.2009.170.1307.

BF08 K. Behrend and B. Fantechi, Symmetric obstruction theories and Hilbert schemes of points on threefolds, Algebra Number Theory 2 (2008), no. 3, 313-345; doi:10.2140/ant.2008.2.313.

BJM19 V. Bussi, D. Joyce, and S. Meinhardt, On motivic vanishing cycles of critical loci, J. Algebraic Geom. 28 (2019), no. 3, 405-438; doi:10.1090/jag/737. 


\section{HALL ALGEBRAS}

BMT14 A. Bayer, E. Macrì, and Y. Toda, Bridgeland stability conditions on threefolds I: BogomolovGieseker type inequalities, J. Algebraic Geom. 23 (2014), no. 1, 117-163; doi:10.1090/S10563911-2013-00617-7.

Bri07 T. Bridgeland, Stability conditions on triangulated categories, Ann. of Math. 166 (2007), no. 2, 317-345; doi:10.4007/annals.2007.166.317.

Bri11_ Hall algebras and curve-counting invariants, J. Amer. Math. Soc. 24 (2011), no. 4, 969-998; doi:10.1090/S0894-0347-2011-00701-7.

Bri12 , An introduction to motivic Hall algebras, Adv. Math. 229 (2012), no. 1, 102-138; doi:10.1016/j.aim.2011.09.003.

Bus14 V. Bussi, Generalized Donaldson-Thomas theory over fields $K \neq \mathbb{C}, 2014$, arXiv:1403.2403.

CDP10 W. Y. Chuang, D. E. Diaconescu, and G. Pan, Rank two ADHM invariants and wallcrossing, Commun. Number Theory Phys. 4 (2010), no. 2, 417-461; doi:10.4310/CNTP.2010.v4.n2.a4.

DM15 B. Davison and S. Meinhardt, Donaldson-Thomas theory for ctegories of homological dimension one with potential, 2015, arXiv:1512.08898.

HL97 D. Huybrechts and M. Lehn, The geometry of moduli spaces of sheaves, Aspects Math., vol. E31 (Friedr. Vieweg \& Sohn, Braunschweig, 1997); doi:10.1007/978-3-663-11624-0.

HRS96 D. Happel, I. Reiten, and S. O. Smalø, Tilting in abelian categories and quasitilted algebras, Mem. Amer. Math. Soc. 120 (1996), no. 575; doi:10.1090/memo/0575.

HT10 D. Huybrechts and R.P. Thomas, Deformation-obstruction theory for complexes via Atiyah and Kodaira-Spencer classes, Math. Ann. 346 (2010), no. 3, 545-569; doi:10.1007/s00208009-0397-6.

Jia16 Y. Jiang, On motivic Joyce-Song formula for the Behrend function identities, 2016, arXiv: 1601.00133.

Jia18_ The Thom-Sebastiani theorem for the Euler characteristic of cyclic $L_{\infty}$-algebras, J. Algebra 498 (2018), 362-397; doi:10.1016/j.jalgebra.2017.11.032.

Joy07 D. Joyce, Configurations in abelian categories. III. Stability conditions and identities, Adv. Math. 215 (2007), no. 1, 153-219; doi:10.1016/j.aim.2007.04.002.

Joy08 Configurations in abelian categories. IV. Invariants and changing stability conditions, Adv. Math. 217 (2008), no. 1, 125-204; doi:10.1016/j.aim.2007.06.011.

Joy15 _ A classical model for derived critical loci, J. Differential Geom. 101 (2015), no. 2, 289-367; doi:10.4310/jdg/1442364653.

JS12 D. Joyce and Y. Song, A theory of generalized Donaldson-Thomas invariants, Mem. Amer. Math. Soc. 217 (2012), no. 1020; doi:10.1090/S0065-9266-2011-00630-1.

KS08 M. Kontsevich and Y. Soibelman, Stability structures, motivic Donaldson-Thomas invariants and cluster transformations, 2008, arXiv:0811.2435.

Lê15 Q. T. Lê, Proofs of the integral identity conjecture over algebraically closed fields, Duke Math. J. 164 (2015), no. 1, 157-194; doi:10.1215/00127094-2869138.

Lan09 A. Langer, Moduli spaces of sheaves and principal G-bundles, Algebraic Geometry, Part 1 (Seattle, 2005), Proc. Sympos. Pure Math., vol. 80 (Amer. Math. Soc., Providence, RI, 2009), 273-308; doi:10.1090/pspum/080.1/2483939.

Li06 J. Li, Zero dimensional Donaldson-Thomas invariants of threefolds, Geom. Topol. 10 (2006), 2117-2171, doi:10.2140/gt.2006.10.2117.

Lie06 M. Lieblich, Moduli of complexes on a proper morphism, J. Algebraic Geom. 15 (2006), no. 1, 175-206; doi:10.1090/S1056-3911-05-00418-2.

Lo12 J. Lo, Polynomial Bridgeland stable objects and reflexive sheaves, Math. Res. Lett. 19 (2012), no. 4, 873-885; doi:10.4310/MRL.2012.v19.n4.a11.

Lo13 Moduli of PT-semistable objects II, Trans. Amer. Math. Soc. 365 (2013), no. 9, 45394573; doi:10.1090/S0002-9947-2013-05622-X. 


\section{Y. TODA}

LP09 M. Levine and R. Pandharipande, Algebraic cobordism revisited, Invent. Math. 176 (2009), no. 1, 63-130; doi:10.1007/s00222-008-0160-8.

MNOP06 D. Maulik, N. Nekrasov, A. Okounkov, and R. Pandharipande, Gromov-Witten theory and Donaldson-Thomas theory. I, Compos. Math. 142 (2006), no. 5, 1263-1285; doi:10.1112/ S0010437X06002302.

Nag10 K. Nagao, On higher rank Donaldson-Thomas invariants, 2010, arXiv:1002.3608.

PP17 R. Pandharipande and A. Pixton, Gromov-Witten/Pairs correspondence for the quintic 3-fold, J. Amer. Math. Soc. 30 (2017), no. 2, 389-449; doi:10.1090/jams/858.

PT09 R. Pandharipande and R.P. Thomas, Curve counting via stable pairs in the derived category, Invent. Math. 178 (2009), no. 2, 407-447; doi:10.1007/s00222-009-0203-9.

PT19 D. Piyaratne and Y. Toda, Moduli of Bridgeland semistable objects on 3-folds and DonaldsonThomas invariants, J. reine angew. Math. 747 (2019), 175-219; doi:10.1515/crelle-20160006.

PTVV13 T. Pantev, B. Toën, M. Vaquié, and G. Vezzosi, Shifted symplectic structures, Publ. Math. Inst. Hautes Études Sci. 117 (2013), 271-328; doi:10.1007/s10240-013-0054-1.

She11 A. Sheshmani, Towards studying of the higher rank theory of stable pairs, Ph.D. thesis, University of Illinois at Urbana-Champaign, 2011; available at https://www.ideals.illinois.edu/ handle/2142/26229.

ST11 J. Stoppa and R.P. Thomas, Hilbert schemes and stable pairs: GIT and derived category wall crossings, Bull. Soc. Math. France 139 (2011), no. 3, 297-339; doi:10.24033/bsmf.2610.

Sto12 J. Stoppa, D0-D6 states counting and GW invariants, Lett. Math. Phys. 102 (2012), no. 2, 149-180; doi:10.1007/s11005-012-0560-y.

Tho00 R.P. Thomas, A holomorphic Casson invariant for Calabi-Yau 3-folds, and bundles on K3 fibrations, J. Differential Geom. 54 (2000), no. 2, 367-438; doi:10.4310/jdg/1214341649.

Tod10a Y. Toda, Curve counting theories via stable objects I. DT/PT correspondence, J. Amer. Math. Soc. 23 (2010), no. 4, 1119-1157; doi:10.1090/S0894-0347-10-00670-3.

Tod10b_ Generating functions of stable pair invariants via wall-crossings in derived categories, New Developments in Algebraic Geometry, Integrable Systems and Mirror Symmetry (RIMS, Kyoto, 2008), Adv. Stud. Pure Math., vol. 59 (Math. Soc. Japan, Tokyo, 2010), 389-434; doi:10.2969/aspm/05910389.

Tod10c On a computation of rank two Donaldson-Thomas invariants, Commun. Number Theory Phys. 4 (2010), no. 1, 49-102; doi:10.4310/CNTP.2010.v4.n1.a2.

Tod11_ Curve counting invariants around the conifold point, J. Differential Geom. 89 (2011), no. $1,133-184$; doi:10.4310/jdg/1324476754.

Tod12 Stability conditions and curve counting invariants on Calabi-Yau 3-folds, Kyoto J. Math. 52 (2012), no. 1, 1-50; doi:10.1215/21562261-1503745.

Tod13a , Bogomolov-Gieseker-type inequality and counting invariants, J. Topol. 6 (2013), no. 1, 217-250; doi:10.1112/jtopol/jts037.

Tod13b_ Curve counting theories via stable objects II: DT/ncDT flop formula, J. reine angew. Math. 675 (2013), 1-51; doi:10.1515/CRELLE.2011.176.

Tod16 Stable pair invariants on Calabi-Yau threefolds containing $\mathbb{P}^{2}$, Geom. Topol. 20 (2016), no. 1, 555-611; doi:10.2140/gt.2016.20.555.

Yukinobu Toda yukinobu.toda@ipmu.jp

Kavli Institute for the Physics and Mathematics of the Universe (WPI), University of Tokyo, 5-1-5 Kashiwanoha, Kashiwa, 277-8583, Japan 